\title{
Extended phase space thermodynamics for third-order Lovelock black holes in diverse dimensions
}

\author{
Hao Xu ${ }^{1, \mathrm{a}}$, Wei $\mathrm{Xu}^{1,2, \mathrm{~b}}$, Liu Zhao ${ }^{1, \mathrm{c}}$ \\ ${ }^{1}$ School of Physics, Nankai University, Tianjin 300071, China \\ ${ }^{2}$ School of Physics, Huazhong University of Science and Technology, Wuhan 430074, China
}

Received: 24 June 2014 / Accepted: 11 September 2014 / Published online: 23 September 2014

(C) The Author(s) 2014. This article is published with open access at Springerlink.com

\begin{abstract}
Treating the cosmological constant as thermodynamic pressure and its conjugate as thermodynamic volume, we investigate the critical behavior of the third-order Lovelock black holes in diverse dimensions. For black hole horizons with different normalized sectional curvature $k=$ $0, \pm 1$, the corresponding critical behaviors differ drastically. For $k=0$, there is no critical point in the extended thermodynamic phase space. For $k=-1$, there is a single critical point in any dimension $d \geq 7$, and for $k=+1$, there is a single critical point in 7 dimensions and two critical points in $8,9,10,11$ dimensions. We studied the corresponding phase structures in all possible cases.
\end{abstract}

\section{Introduction}

The thermodynamics of a black hole has been a research frontier for several decades. In the presence of a negative cosmological constant, there can be very rich phase structures in the black hole thermodynamic phase space. Since the early work [1] on the phase transition in the Schwarzschild AdS black hole, which is presently known as a Hawking-Page transition, our understanding as regards black hole phase transitions has been greatly extended. An important example is the first-order phase transition in Reissner-Nordström-AdS (RN-AdS) spacetime [2-4], which has been compared with Van der Waals liquid-gas phase transition frequently.

Recently, the idea of including the cosmological constant in the first law of black hole has become popular [5-8]. Following this idea, the cosmological constant is no longer a fixed parameter, but rather a thermodynamic variable. The AdS background can be varying. One may doubt the neces-

\footnotetext{
${ }^{a}$ e-mail: physicshx@gmail.com

b e-mail: xuweifuture@mail.nankai.edu.cn

ce-mail: lzhao@nankai.edu.cn
}

sity of this consideration. However, there are indeed some physical reasons for doing so [9-12]. Under this consideration, the black hole mass should be identified as the enthalpy $H$ rather than the internal energy [5], and the cosmological constant becomes an effective thermodynamic pressure,

$P=-\frac{\Lambda}{8 \pi}$.

The thermodynamic volume $V$ that is conjugate to $P$ is naturally defined as $V=\left(\frac{\partial H}{\partial P}\right)_{S}$. A detailed study of the volume can be found in [13]. The temperature of the black hole is a function of the black hole radius (which is closely related to $V)$ and the cosmological constant. Such a relationship can be inverted and taken as the equation of states (EOS) for the black hole system, and hence one can adopt the usual methods used in classical thermodynamics to analyze the critical behavior of the black hole.

There have been many papers pursuing the above idea for diverse choices of AdS black holes, and most works indicate that there is a close analogy between the $P-V$ criticalities of AdS black holes and the phase transition in Van der Waals liquid-gas system. Reference [9] is an investigation of the 4dimensional RN AdS black hole in the extended phase space, which proved that the analogy with Van der Waals system is very precise. Then the analogy has been extended to other cases, including higher dimensional charged black holes [9, $14,15]$, rotating black holes and black rings [16-20], GaussBonnet black holes [21-23], $f(R)$ black holes [24], black holes with scalar hair [25,26], black holes with nonlinear source [27], Born-Infeld black holes [28,29], RN de Sitter black holes [30], and the third-order Lovelock-Born-Infeld black holes in $d=7$ [31]. In a recent work [32], we studied the criticality of static Gauss-Bonnet black holes in AdS spacetime, taking the Gauss-Bonnet coupling constant as a free thermodynamic variable. See [33-36] for some related work. 
In this work we shall study the $P-V$ criticality of the static black holes in the third-order Lovelock gravity in diverse dimensions. In the presence of a cosmological constant, the black holes can be classified using the normalized sectional curvature of the black hole horizons. There are three different classes of black hole solutions in this classification scheme, i.e. black holes with horizon curvatures $k=0, \pm 1$.

For $k=0$, the EOS is identical to that of an ideal gas, thus no phase transition could occur. For $k=+1$ and $d=7$, there is one critical point and the first-order phase transition can easily be obtained. All these results are the same as in the work of [31]. Furthermore, we give a detailed analysis of $k=$ \pm 1 and $d \geq 7$. For $k=-1$, there is one critical point in any dimension $d \geq 7$. When $v=v_{c}$ the system is physical if and only if $P=P_{c}$. Actually all the isobaric plots of temperature diverge at $v=v_{c}$, except the one corresponding to $P=P_{c}$. We can find the first-order phase transition at the isobaric plots of the Gibbs free energy in specific regions of $P$. When $k=+1$, the situation is a little more complicated. There is one critical point in 7 dimensions, there are two critical points in $8,9,10,11$ dimensions, and no critical points in $d \geq 12$ dimensions.

The paper is organized as follows. In the next section we will give a brief review of the thermodynamics of the thirdorder Lovelock black holes. In Sect. 3 we give the EOS and find the critical points in diverse dimensions. In Sect. 4 we investigate the critical behavior of the system. Finally in Sect. 5 we present some concluding remarks.

\section{Thermodynamics of third-order Lovelock black holes}

To start with we give a brief review of the thermodynamics of the third-order Lovelock black holes [37-39]. Setting the Newton constant $G=1$, the action is given by

$\mathcal{I}=\frac{1}{16 \pi} \int \mathrm{d}^{d} x \sqrt{-g}\left(R-2 \Lambda+\alpha_{2} \mathcal{L}_{2}+\alpha_{3} \mathcal{L}_{3}\right)$

where the Gauss-Bonnet and the third-order Lovelock densities are given as

$$
\begin{aligned}
\mathcal{L}_{2}= & R_{\mu \nu \gamma \delta} R^{\mu \nu \gamma \delta}-4 R_{\mu \nu} R^{\mu \nu}+R^{2} \\
\mathcal{L}_{3}= & R^{3}+2 R^{\mu \nu \sigma \kappa} R_{\sigma \kappa \rho \tau} R_{\mu \nu}^{\rho \tau} \\
& +8 R_{\sigma \rho}^{\mu \nu} R_{\nu \tau}^{\sigma \kappa} R_{\mu \kappa}^{\rho \tau}+24 R^{\mu \nu \sigma \kappa} R_{\sigma \kappa \nu \rho} R_{\mu}^{\rho} \\
& +3 R R^{\mu \nu \sigma \kappa} R_{\mu \nu \sigma \kappa}+24 R^{\mu \nu \sigma \kappa} R_{\sigma \mu} R_{\kappa \nu} \\
& +16 R^{\mu \nu} R_{\nu \sigma} R_{\mu}^{\sigma}-12 R R^{\mu \nu} R_{\mu \nu},
\end{aligned}
$$

$\alpha_{2}$ and $\alpha_{3}$, respectively, are the second (i.e. Gauss-Bonnet) and the third Lovelock coefficients. For the particular choice of Gauss-Bonnet and Lovelock coefficients

$\alpha_{2}=\frac{\alpha}{(d-3)(d-4)}, \quad \alpha_{3}=\frac{\alpha^{2}}{72\left(\begin{array}{c}d-3 \\ 4\end{array}\right)}$,

it is well known that there exists an analytic static black hole solution of the form [37-39]

$$
\begin{aligned}
& d s^{2}=-f(r) \mathrm{d} t^{2}+\frac{1}{f(r)} \mathrm{d} r^{2}+r^{2} \mathrm{~d} \Omega_{k}^{2}, \\
& f(r)=k+\frac{r^{2}}{\alpha}\left[1-\left(1+\frac{6 \Lambda \alpha}{(d-1)(d-2)}+\frac{3 \alpha m}{r^{d-1}}\right)^{\frac{1}{3}}\right],
\end{aligned}
$$

where $k=0, \pm 1$ if $\Lambda<0$ and $k=+1$ if $\Lambda \geq 0, d \Omega_{k}^{2}$ is the line element on a $(d-2)$-dimensional maximally symmetric Einstein manifold with curvature $k$. We will be working mostly with $\Lambda<0$; however, we shall see that $P=-\frac{\Lambda}{8 \pi}$ can become negative (i.e. $\Lambda$ can become positive) in an isothermal process. The same phenomenon has also been observed while studying the $P-V$ criticalities of other AdS black holes, see e.g. in [28] for the case of RN-AdS black hole. Since the $P-V$ analysis may not be valid for $\Lambda>0$, we consider the case $\Lambda>0$ as unphysical in our paper.

The gravitational mass $M$ can be expressed as $\frac{(d-2) \Sigma_{k}}{16 \pi G} m$, where $\Sigma_{k}$ is the volume of the $(d-2)$-dimensional submanifold just mentioned. The radius $r_{+}$of the black hole is one of the roots of $f(r)$ (in AdS spacetime, it is the largest root of $f(r))$. Identifying $H \equiv M$ we can rearrange the equations $f\left(r_{+}\right)=0$ and $T=\frac{f^{\prime}\left(r_{+}\right)}{4 \pi}$ in the form

$H=\frac{(d-2) \Sigma_{k} r_{+}^{d-3}}{16 \pi}\left(k+\frac{16 \pi P r_{+}^{2}}{(d-1)(d-2)}+\frac{\alpha k^{2}}{r_{+}^{2}}+\frac{\alpha^{2} k}{3 r_{+}^{4}}\right)$,

$$
\begin{aligned}
T= & \frac{1}{12 \pi r_{+}\left(r_{+}^{2}+k \alpha\right)^{2}}\left[\frac{48 \pi r_{+}^{6} P}{(d-2)}\right. \\
& \left.+3(d-3) r_{+}^{4} k+3(d-5) r_{+}^{2} \alpha k^{2}+(d-7) \alpha^{2} k\right] .
\end{aligned}
$$

Among various choices for the spacetime dimension $d$, the particular case $d=7$ is qualitatively different from other choices, because the last term in (9) vanishes when $d=7$. Consequently, the temperature $T$ vanishes as $r_{+} \rightarrow 0$ when $d=7$, while it becomes divergent as $r_{+} \rightarrow 0$ in higher dimensions. That the case $d=7$ is distinguished from the cases of higher dimensions is perhaps a consequence of the fact that $d=7$ is the lowest dimension in which the thirdorder Lovelock density can affect the local geometry. We shall see later that the critical behavior in $d=7$ is also distinguished from the cases of higher dimensions. 
The other thermodynamic quantities which we need in the following discussions are given as follows. These are the black hole entropy [39]

$S=\frac{\Sigma_{k} r_{+}^{d-2}}{4}\left[1+\frac{2(d-2) k \alpha}{(d-4) r_{+}^{2}}+\frac{(d-2) k^{2} \alpha^{2}}{(d-6) r_{+}^{4}}\right]$

and the thermodynamic volume

$V=\left(\frac{\partial H}{\partial P}\right)_{S, \alpha}=\frac{r^{d-1} \Sigma_{k}}{d-1}$

We see that the thermodynamic volume is a monotonic function of the radius of the horizon. The first law of black hole thermodynamics in the extended phase space can be expressed as

$\mathrm{d} H=T \mathrm{~d} S+V \mathrm{~d} P+\psi \mathrm{d} \alpha$,

where $\psi$ is the thermodynamic conjugate of $\alpha$, which is given by

$\psi=\left(\frac{\partial H}{\partial \alpha}\right)_{S, P}=\frac{\Sigma_{k} r_{+}^{d-7} k^{2}}{48 \pi}\left(2 k \alpha-3 r_{+}^{2}\right)(d-2)$.

It should be remarked that in general cases the first law should contain contributions from the Gauss-Bonnet and Lovelock coefficients as independent thermodynamic variables. However, in our case, these two objects are proportional to each other and we are left with only a single parameter $\alpha$ as given in (5). A detailed discussion of extended first Law and Smarr formula for Lovelock gravity can be found in [40].

The Gibbs free energy can be obtained by

$$
\begin{aligned}
G= & G(T, P)=H-T S \\
= & \Sigma_{k}\left\{\frac{r_{+}^{d-1} P}{d-1}+\frac{(d-2)\left(k^{2} \alpha^{2}+3 r_{+}^{2} k \alpha+3 r_{+}^{4}\right) k r_{+}^{d-7}}{48 \pi}\right. \\
& -\frac{r_{+}^{d-7}}{48 \pi\left(r_{+}^{2}+k \alpha\right)^{2}}\left(\frac{r_{+}^{4}}{d-2}+\frac{2 k \alpha r_{+}^{2}}{d-4}+\frac{k^{2} \alpha^{2}}{d-6}\right) \\
& \times\left(48 r_{+}^{6} \pi P+(d-2)\left[3 k(d-3) r_{+}^{4}+3 k^{2} \alpha(d-5) r_{+}^{2}\right.\right. \\
& \left.\left.\left.+\alpha^{2} k^{3}(d-7)\right]\right)\right\} .
\end{aligned}
$$

Notice that although on the left hand side we have included $T$ and $P$ as independent variables for the Gibbs free energy, the right hand side does not explicitly contain $T$. To understand Eq. (14), we must think of $r_{+}$as an implicit function of $T$ and $P$. The implicit relationship is given by the expression (9) for the temperature.

\section{Equation of states and critical points}

Equation (9) can be rearranged into the following form:

$$
\begin{aligned}
P= & \frac{T(d-2)}{4 r_{+}}-\frac{k(d-2)(d-3)}{16 \pi r_{+}^{2}}+\frac{T k \alpha(d-2)}{2 r_{+}^{3}} \\
& -\frac{k^{2} \alpha(d-2)(d-5)}{16 \pi r+^{4}} \\
& +\frac{T k^{2} \alpha^{2}(d-2)}{4 r_{+}^{5}}-\frac{k^{3} \alpha^{2}(d-2)(d-7)}{\pi r_{+}^{6}} .
\end{aligned}
$$

This equation can be regarded as the thermodynamic EOS for the black hole system. Instead of the thermodynamic volume $V$, we introduce the parameter

$v=\frac{4 r_{+}}{d-2}$

as an effective specific volume. Then the EOS takes the form

$$
\begin{aligned}
P= & \frac{T}{v}-\frac{k(d-3)}{(d-2) \pi v^{2}}+\frac{32 T k \alpha}{(d-2)^{2} v^{3}}-\frac{16 k^{2} \alpha(d-5)}{(d-2)^{3} \pi v^{4}} \\
& +\frac{256 T k^{2} \alpha^{2}}{(d-2)^{4} v^{5}}-\frac{256 k^{3} \alpha^{2}(d-7)}{3(d-2)^{5} \pi v^{6}}
\end{aligned}
$$

which resembles the EOS of the Van der Waals system to some extent.

The critical points, if they exist, correspond to inflection points on the isotherms, i.e. they must obey the conditions

$\frac{\partial P}{\partial v}=0, \quad \frac{\partial^{2} P}{\partial^{2} v}=0$

and $\frac{\partial^{2} P}{\partial^{2} v}$ should change sign around each of the solutions. Merely finding the solution of (18) is insufficient to justify the existence of a critical point, because the second derivative $\frac{\partial^{2} P}{\partial^{2} v}$ may not change sign around the solution, making the solution correspond to an extremum, rather than an inflection point. Later we shall see that when $k=1$ and $d=12$, the solution to (18) is indeed not an inflection point and thus no critical point exists in 12 dimensions.

Now let us proceed to finding all possible critical points for each value of $k=0, \pm 1$ in diverse dimensions.

\subsection{Ricci flat case with $k=0$}

When $k=0$, Eq. (17) reduces into

$P=\frac{T}{v}$

This equation is independent of the spacetime dimension $d$ and is identical to the EOS of an ideal gas. The conditions 
(18) regarded as a system of algebraic equations for $T$ and $v$ have no finite nonzero solution, so there is no critical point when $k=0$ in any dimension.

\subsection{Hyperbolic case with $k=-1$}

In this case, Eq. (17) becomes

$$
\begin{aligned}
P= & \frac{T}{v}+\frac{(d-3)}{(d-2) \pi v^{2}}-\frac{32 T \alpha}{(d-2)^{2} v^{3}}-\frac{16 \alpha(d-5)}{(d-2)^{3} \pi v^{4}} \\
& +\frac{256 T \alpha^{2}}{(d-2)^{4} v^{5}}+\frac{256 \alpha^{2}(d-7)}{3(d-2)^{5} \pi v^{6}} .
\end{aligned}
$$

Correspondingly, Eq. (18) possesses a single solution:

$v_{c}=\frac{4 \sqrt{\alpha}}{d-2}$

$T_{c}=\frac{1}{2 \pi \sqrt{\alpha}}$

which can be checked to be a real critical point. The corresponding critical pressure is

$P_{c}=P\left(v_{c}, T_{c}\right)=\frac{1}{48 \pi \alpha}(d-1)(d-2)$.

The critical parameters $v_{c}$ and $T_{c}$ must all be real positive, so the existence of a critical point requires $\alpha>0$. It is easy to check that

$$
\frac{P_{c} v_{c}}{T_{c}}=\frac{d-1}{6},
$$

which depends only on $d$ but not on any other parameters. Remember that the above critical point exists in all dimensions $d \geq 7$.

\subsection{Spherical case with $k=1$}

In this case the EOS reads

$$
\begin{aligned}
P= & \frac{T}{v}-\frac{(d-3)}{(d-2) \pi v^{2}}+\frac{32 T \alpha}{(d-2)^{2} v^{3}}-\frac{16 \alpha(d-5)}{(d-2)^{3} \pi v^{4}} \\
& +\frac{256 T \alpha^{2}}{(d-2)^{4} v^{5}}-\frac{256 \alpha^{2}(d-7)}{3(d-2)^{5} \pi v^{6}} .
\end{aligned}
$$

We have two solutions to Eq. (18), which read

$$
\begin{aligned}
v_{c 1} & =\frac{4 \sqrt{\alpha}}{d-2}\left(\frac{d+3-2 \mathcal{A}}{d-3}\right)^{1 / 2}, \\
T_{c 1} & =\frac{d-3}{2 \pi \sqrt{\alpha}}\left(\frac{\mathcal{A}-d+2}{\mathcal{A}-3 d+6}\right)\left(\frac{d-3}{d+3-2 \mathcal{A}}\right)^{1 / 2},
\end{aligned}
$$

Table 1 The numerical critical parameters at the first critical point

\begin{tabular}{lrllr}
\hline Dimension $d$ & \multicolumn{1}{c}{$P_{c 1}$} & $v_{c 1}$ & $T_{c 1}$ & $\frac{P_{c 1} v_{c 1}}{T_{c 1}}$ \\
\hline 8 & -0.4336 & 0.3269 & 0.1364 & -1.0392 \\
9 & -0.0856 & 0.3928 & 0.2046 & -0.1644 \\
10 & 0.0374 & 0.4226 & 0.2636 & 0.0600 \\
11 & 0.1194 & 0.4444 & 0.3183 & 0.1667
\end{tabular}

Table 2 The numerical critical parameters at the second critical point

\begin{tabular}{lllll}
\hline Dimension $d$ & $P_{c 2}$ & $v_{c 2}$ & $T_{c 2}$ & $\frac{P_{c 2} v_{c 2}}{T_{c 2}}$ \\
\hline 7 & 0.0271 & 1.7889 & 0.1424 & 0.3400 \\
8 & 0.0454 & 1.3597 & 0.1857 & 0.3325 \\
9 & 0.0696 & 1.0732 & 0.2302 & 0.3244 \\
10 & 0.1002 & 0.8660 & 0.2757 & 0.3148 \\
11 & 0.1383 & 0.7027 & 0.3221 & 0.3017 \\
\hline
\end{tabular}

and

$v_{c 2}=\frac{4 \sqrt{\alpha}}{d-2}\left(\frac{d+3+2 \mathcal{A}}{d-3}\right)^{1 / 2}$,

$T_{c 2}=\frac{d-3}{2 \pi \sqrt{\alpha}}\left(\frac{\mathcal{A}+d-2}{\mathcal{A}+3 d-6}\right)\left(\frac{d-3}{d+3+2 \mathcal{A}}\right)^{1 / 2}$,

where

$\mathcal{A}=\sqrt{(d-2)(12-d)}$.

The corresponding pressures are given, respectively, as follows:

$$
\begin{aligned}
& P_{c 1} \\
& =\frac{(d-2)(d-3)^{2}\left[\left(15 d^{2}-222 d+177\right) d-\mathcal{A}\left(d^{2}-160 d+255\right)+414\right]}{48 \pi \alpha(d+3-2 \mathcal{A})^{3}(\mathcal{A}-3 d+6)},
\end{aligned}
$$

$$
\begin{aligned}
& P_{c 2} \\
& =-\frac{(d-2)(d-3)^{2}\left[\left(15 d^{2}-222 d+177\right) d+\mathcal{A}\left(d^{2}-160 d+255\right)+414\right]}{48 \pi \alpha(d+3+2 \mathcal{A})^{3}(\mathcal{A}+3 d-6)} .
\end{aligned}
$$

For these solutions to be real-valued, the constant $\mathcal{A}$ must also be real-valued. This requires $2 \leq d \leq 12$. On the other hand, the third-order Lovelock density is geometrically nontrivial only when $d \geq 7$, therefore, for $k=+1$, critical points can only possibly exist in dimensions $7 \leq d \leq 12$. In particular, when $d=12$, the above two solutions degenerate, and one can check that $\frac{\partial^{2} P}{\partial^{2} v}$ does not change sign around this degenerate solution. When $d=7$, both $v_{c 1}$ and $T_{c 1}$ vanish, with the corresponding $P_{c 1}$ going to negative infinity. Clearly $v_{c 1}=0$ does not correspond to a black hole configuration, so the first 


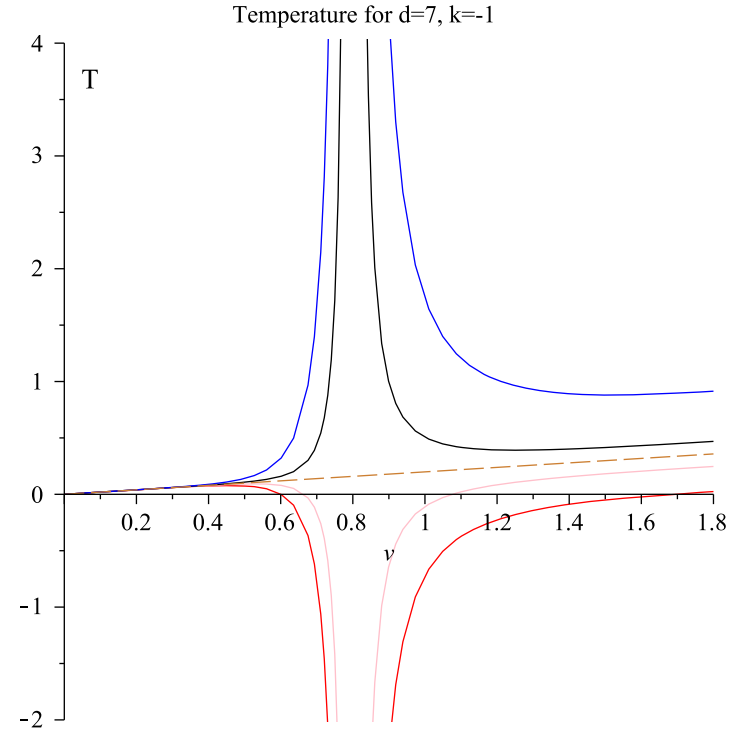

Fig. 1 The isobaric (left) and isothermal (right) plots at $d=7, k=$ -1 . On the left plots, all the isobars are discontinuous at $v=v_{c}$, except the one corresponding to $P=P_{c}$ (dashed line), and the pressure decreases from top to bottom. Similarly, on the right plots, all the

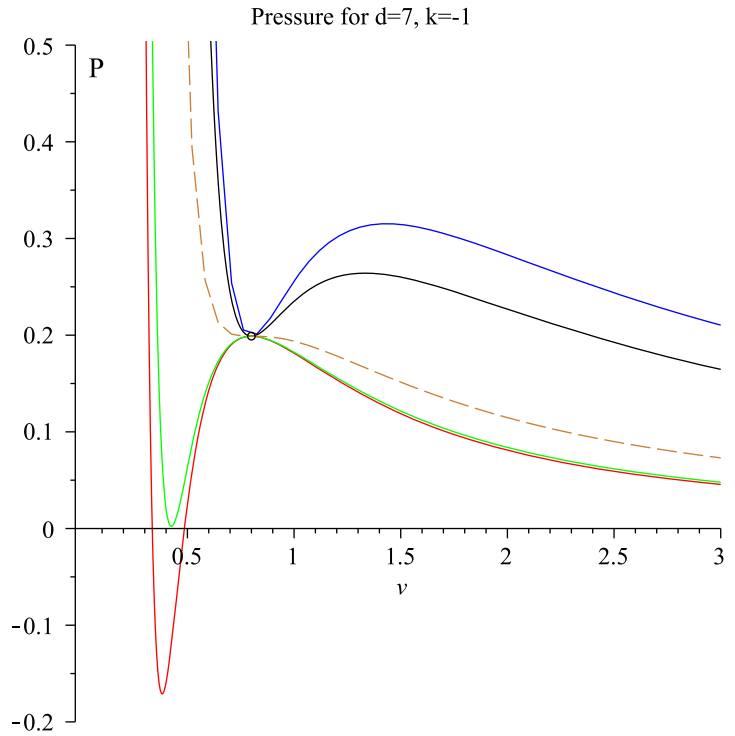

isotherms are discontinuous at $v=v_{c}$, except the dashed line corresponding to $T=T_{c}$. The temperatures decrease from top to bottom on the right plots
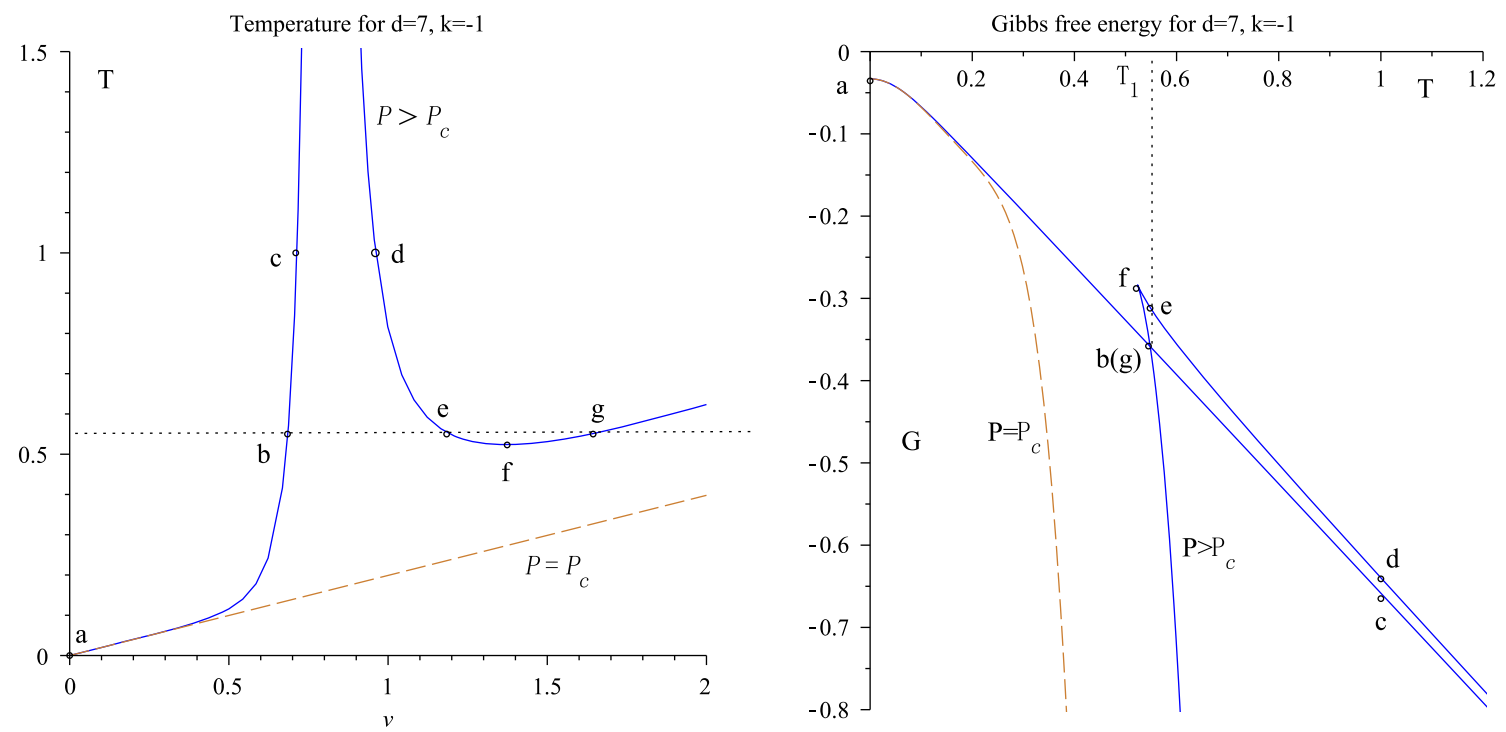

Fig. $2 d=7$ and $k=-1$ : isobaric plots of the EOS and Gibbs free energy at $P=0.2785>P_{c}$. For reference, the isobaric curves at $P=P_{c}$ are also depicted in dashed lines. Marked points on the left and right diagrams are in one-to-one correspondence

solution is excluded from the possible candidates of critical points when $d=7$. One can check that for $8 \leq d \leq 11$, $\frac{\partial^{2} P}{\partial^{2} v}$ indeed changes sign around each of the above solutions, and for $d=7$, the above object also changes sign around the second solution. So we conclude that, when $k=+1$, there will be a single critical point in dimensions $d=7$ and two critical points in dimensions $d=8,9,10,11$.
It is trivial to show that the combinations

$$
\frac{P_{c 1} v_{c 1}}{T_{c 1}}=\frac{\left(177-222 d+15 d^{2}\right) d-\mathcal{A}\left(d^{2}-160 d+255\right)+414}{6(2 \mathcal{A}-d-3)^{2}(\mathcal{A}-d+2)},
$$

$\frac{P_{c 2} v_{c 2}}{T_{c 2}}=-\frac{\left(177-222 d+15 d^{2}\right) d+\mathcal{A}\left(d^{2}-160 d+255\right)+414}{6(2 \mathcal{A}+d+3)^{2}(\mathcal{A}+d-2)}$ 

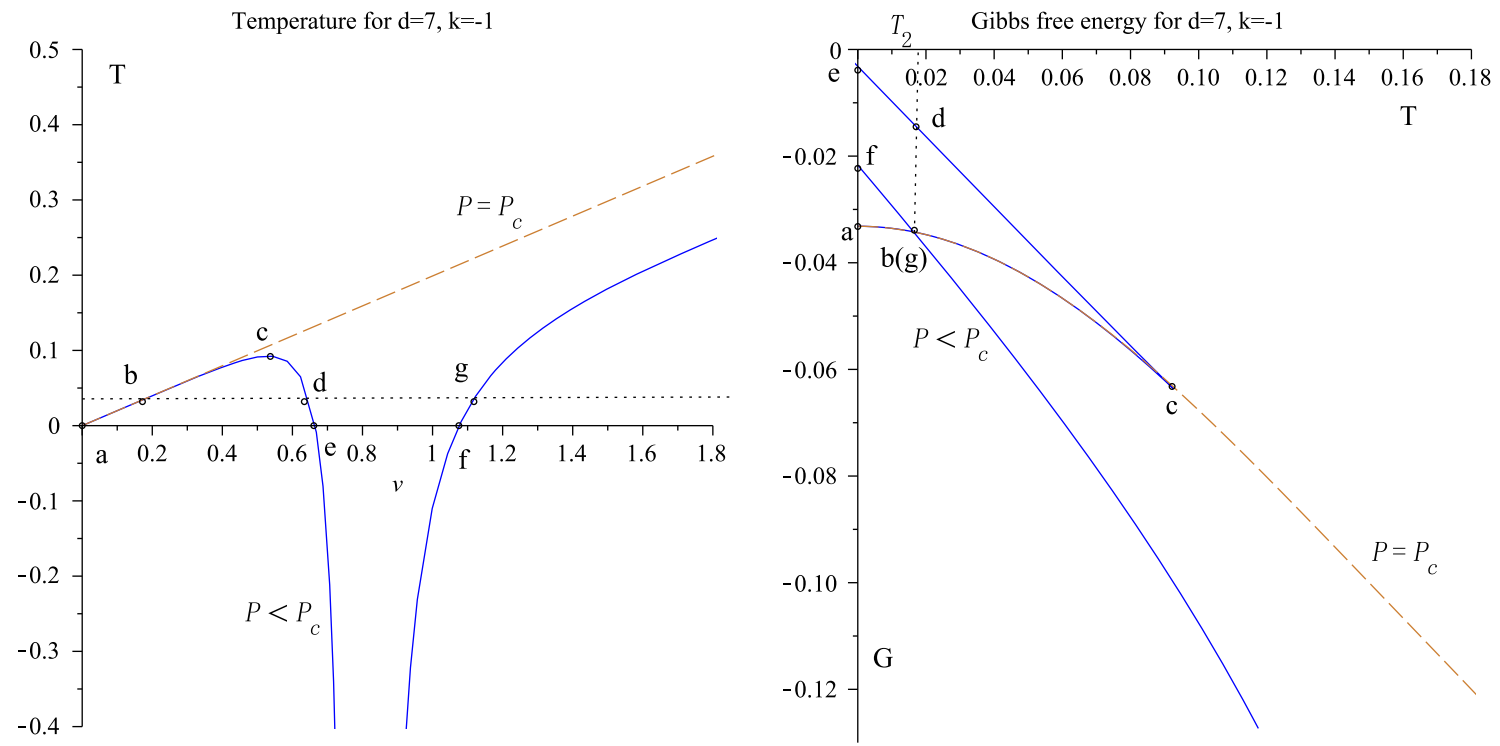

Fig. $3 d=7$ and $k=-1$ : isobaric plots of the EOS and Gibbs free energy at $P=0.1592<P_{c}$. For reference, the isobaric curves at $P=P_{c}$ are also depicted in dashed lines. Marked points on the left and right diagrams are in one-to-one correspondence
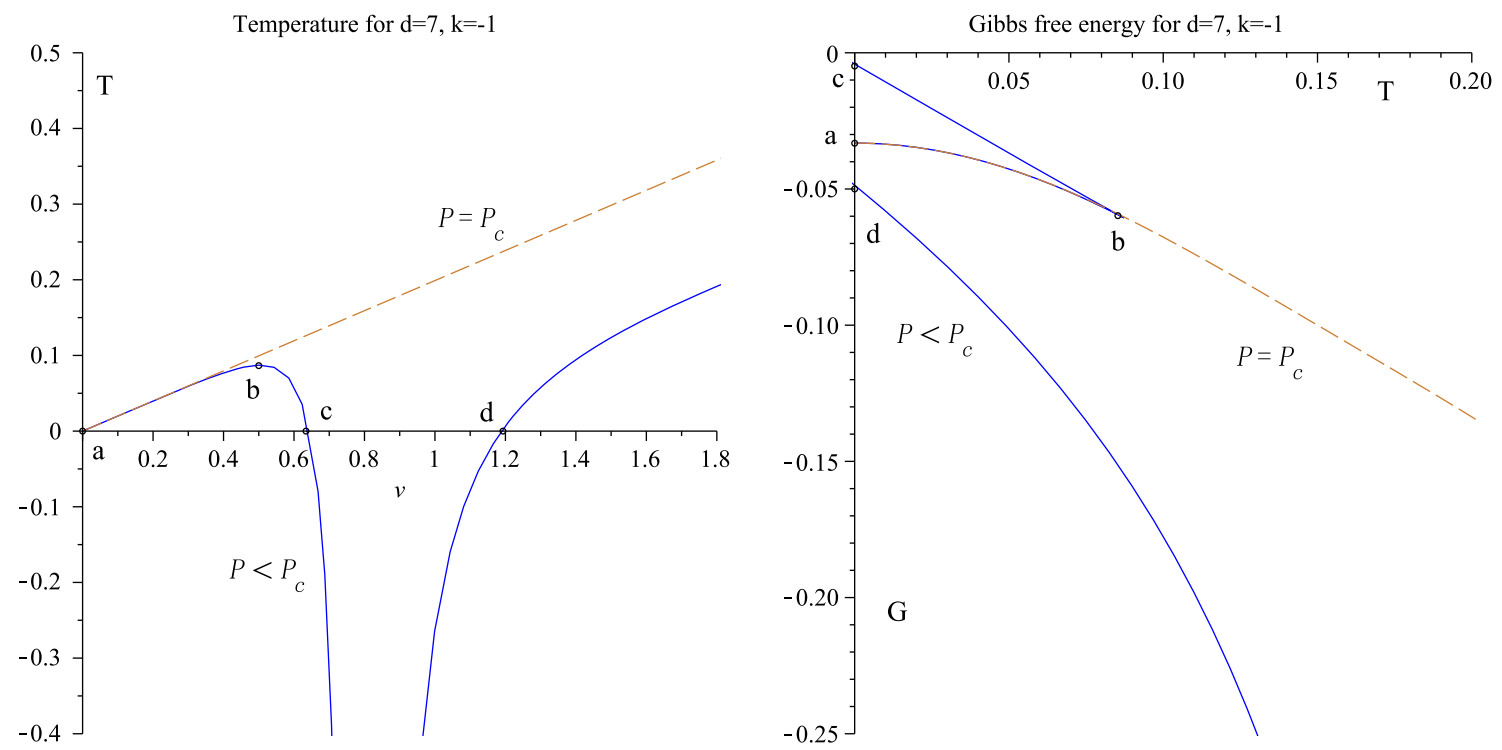

Fig. $4 d=7$ and $k=-1$ : isobaric plots of the EOS and Gibbs free energy at $P=0.1393<P_{c}$. For reference, the isobaric curves at $P=P_{c}$ are also depicted in dashed lines. Marked points on the left and right diagrams are in one-to-one correspondence

are both only dependent on the dimension $d$. The numerical values for the critical parameters are given in Tables 1 and 2. The values for $P_{c}$ are given in units of $\alpha^{-1}$, those for $v_{c}$ are given in units of $\alpha^{1 / 2}$ and for $T_{c}$ are given in units of $\alpha^{-1 / 2}$. In all subsequent discussions we will stick to this system of units. The critical pressure $P_{c 1}$ is negative in dimensions $d=8,9$. Although the black hole solutions remain valid for a positive cosmological constant, we consider them as unphysical in this paper.

\section{Phase structures}

\subsection{Hyperbolic case with $k=-1$}

Previous studies indicate that critical points may exist for AdS black holes with $k=-1$ in various models of gravity. However, the behavior of such black holes near the criticalities is in some sense exotic and has been paid less attention to as compared to the cases of $k=+1$ black holes. In this 

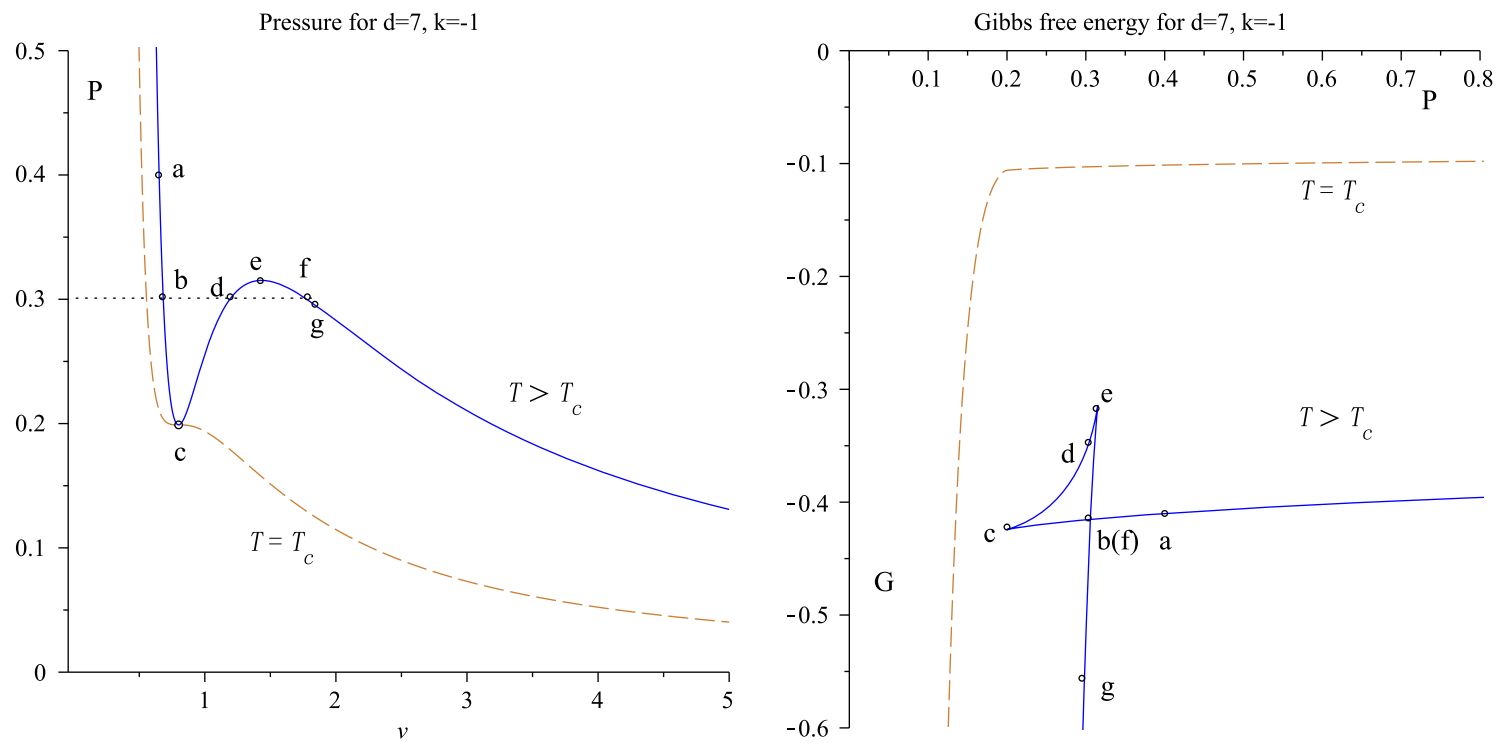

Fig. $5 d=7$ and $k=-1$ : isothermal plots of the EOS and Gibbs free energy at $T=0.6366>T_{c}$. For reference, the isothermal curves at $T=T_{c}$ are also depicted in dashed lines. Marked points on the left and right diagrams are in one-to-one correspondence
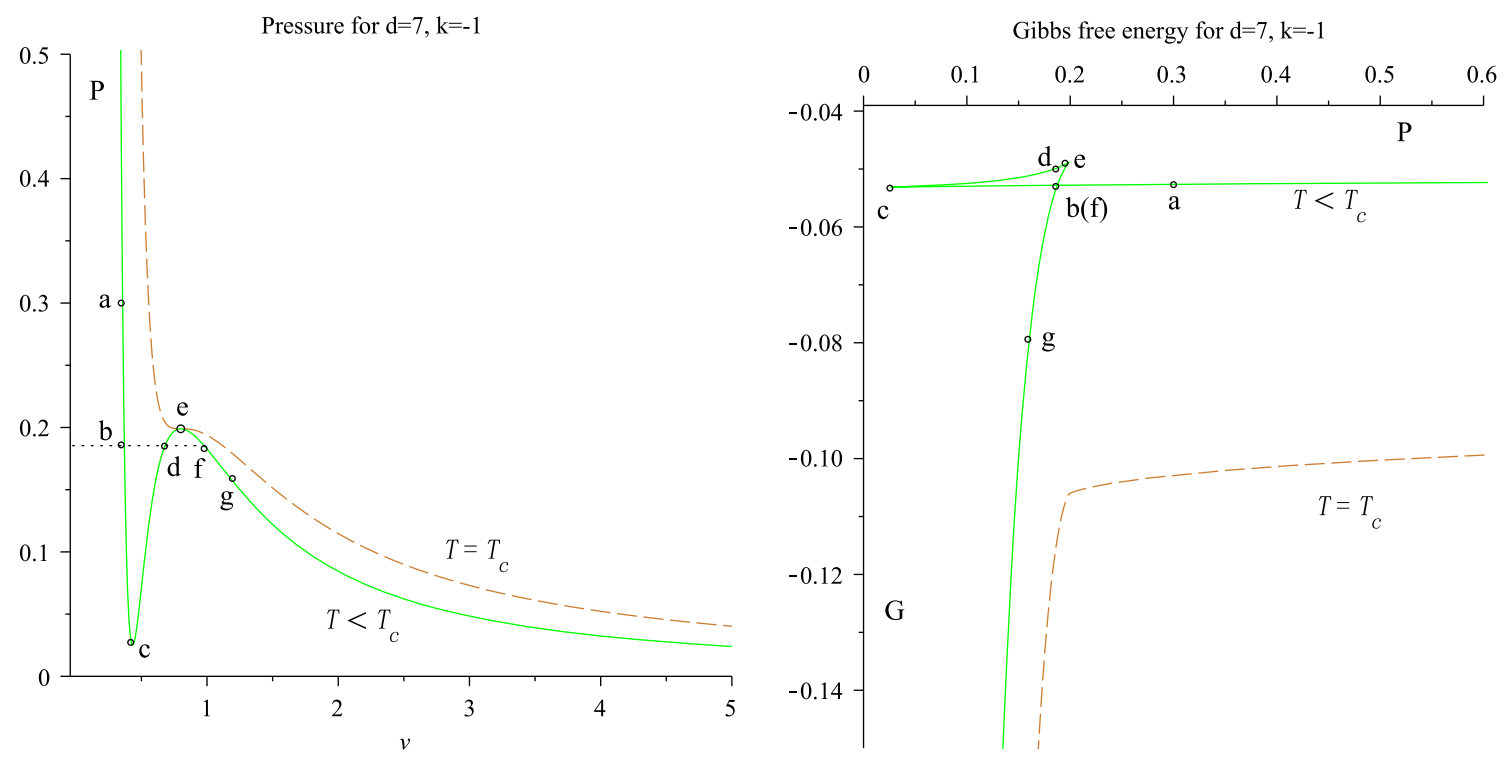

Fig. $6 d=7$ and $k=-1$ : isothermal plots of the EOS and Gibbs free energy at $T=0.0732<T_{c}$. For reference, the isothermal curves at $T=T_{c}$ are also depicted in dashed lines. Marked points on the left and right diagrams are in one-to-one correspondence

work, we will pay particular attention to the $k=-1$ cases and show how exotic it is for such black holes.

As mentioned earlier, the Hawking temperature (9) (and hence the EOS) is qualitatively different for $d=7$ and $d>7$, so we shall subdivide our discussions into $d=7$ and $d>7$ cases.

\subsubsection{The case of $d=7$}

In the rest of the paper, we will treat the thermodynamics of the Lovelock AdS black holes as a $P-V-T$ system, taking the Lovelock coefficient $\alpha$ as a constant parameter. This is of course an incomplete description of the Lovelock black holes, because the coupling coefficient $\alpha$ should also play a role in the thermodynamics of the black holes, just like in the case of GB AdS black holes [32].

Figure 1 gives the isobaric and isothermal plots for our $P-$ $V-T$ system at $d=7$ and $k=-1$, in which the parameter $\alpha$ is taken to be equal to 1 . The critical value of the parameters are $P_{c}=\frac{5}{8 \pi}, v_{c}=\frac{4}{5}$, and $T_{c}=\frac{1}{2 \pi}$.

From the isobaric plots given in Fig. 1, one can see that for each $P<P_{c}$, there is a region for $v$ containing $v_{c}$ 

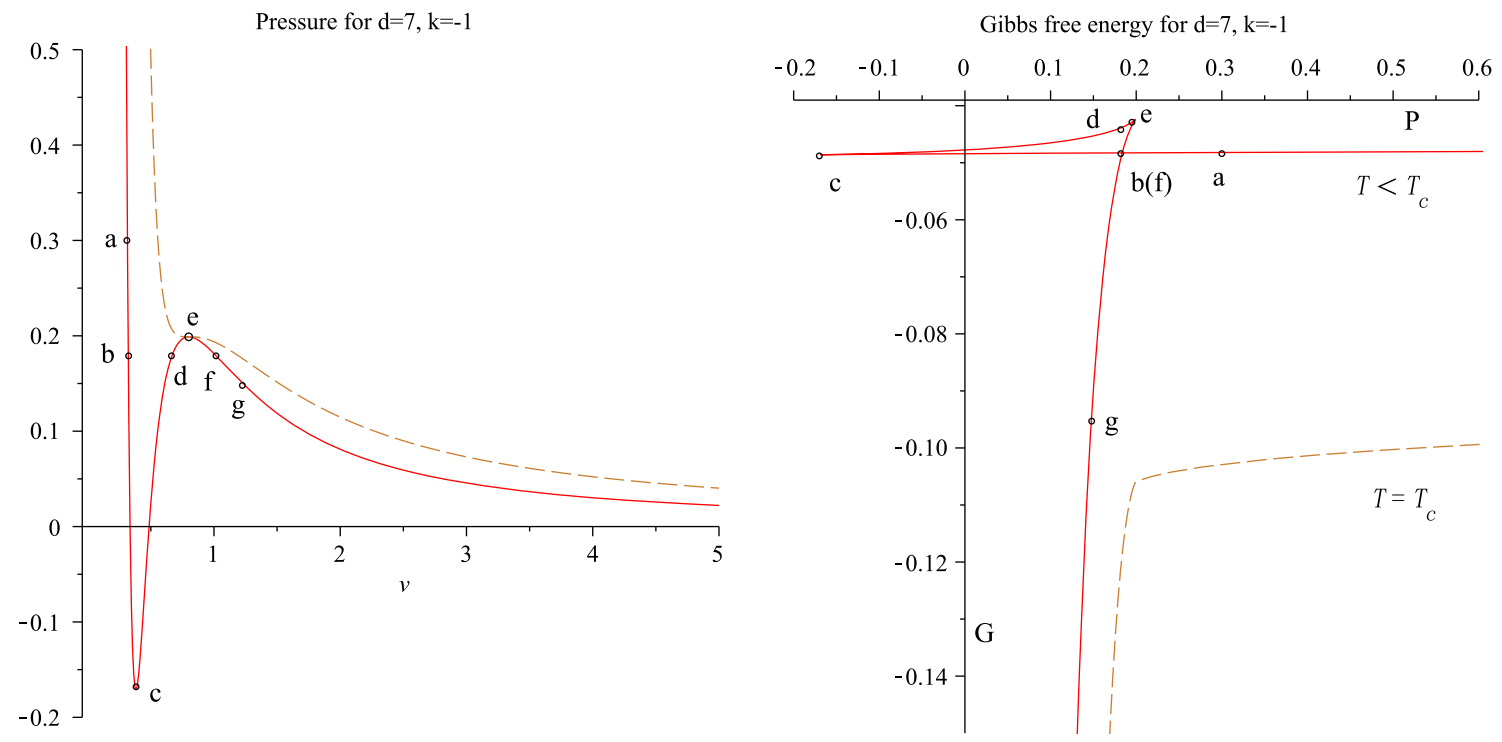

Fig. $7 d=7$ and $k=-1$ : isothermal plots of the EOS and Gibbs free energy at $T=0.0637<T_{c}$. For reference, the isothermal curves at $T=T_{c}$ are also depicted in dashed lines. Marked points on the left and right diagrams are in one-to-one correspondence

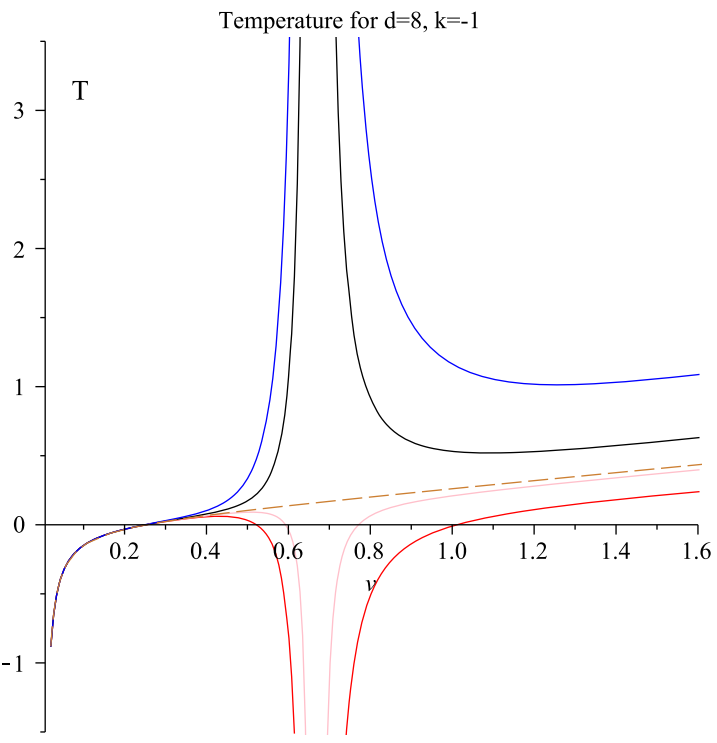

Fig. 8 The isobaric (left) and isothermal (right) plots at $d=8, k=$ -1 . On the left plots, all the isobars are discontinuous at $v=v_{c}$, except the one corresponding to $P=P_{c}$ (dashed line), and the pressure decreases from top to bottom. On the right plots, all isotherms are dis-

such that the temperature $T$ goes negative, which means that black holes with such parameters are thermodynamically unstable and should not exist actually. Meanwhile, on the isothermal plots, one sees that for sufficiently low temperatures $T<T_{c}$, there is a segment on the isotherms such that the pressure becomes negative. Such black hole states are physically impossible, because negative $P$ corresponds to positive cosmological constant $\Lambda$, and it is well known

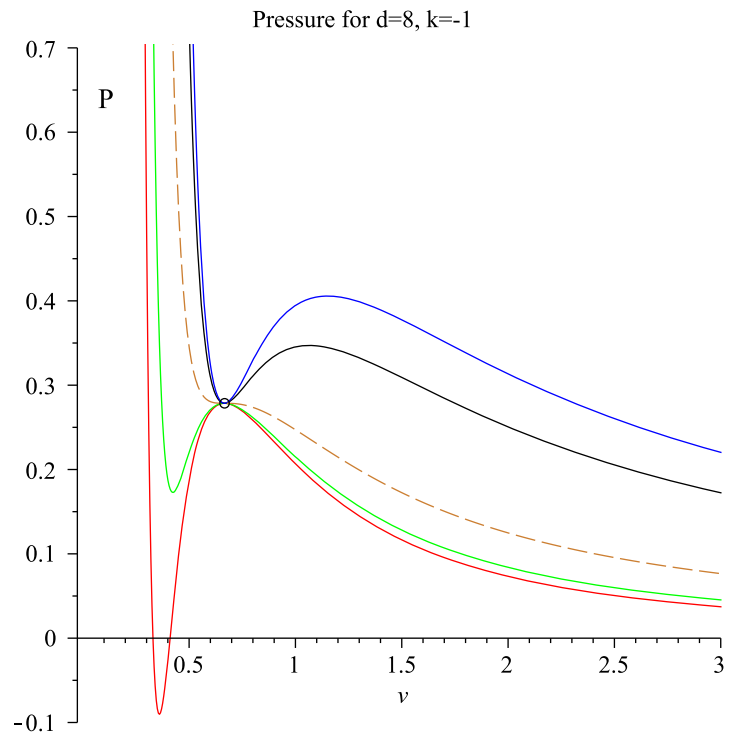

continuous at $v=v_{c}$, except the dashed one corresponding to $T=T_{c}$. The temperature of each isotherm decreases from top to bottom on the right plots

that black holes with positive cosmological constant cannot have $k=-1$.

To have more intuitive feelings on the critical behavior of the $d=7, k=-1$ black holes, let us turn to look at the Gibbs free energy plots. Since $G=G(T, P)$, we will consider separately isobaric and isothermal processes.

The isobaric plots of the EOS and Gibbs free energy are presented respectively for black holes with constant $P>P_{c}$ 

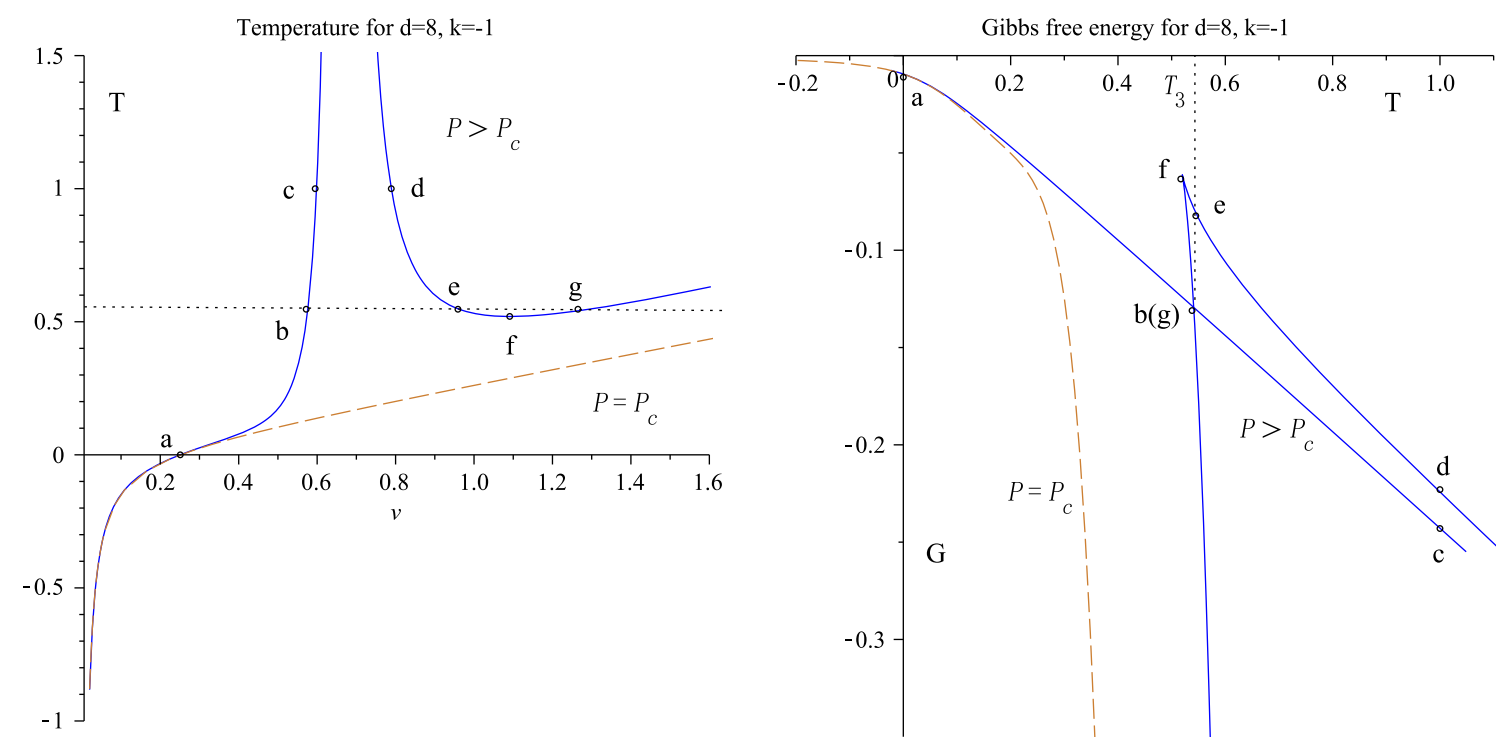

Fig. $9 d=8$ and $k=-1$ : isobaric plots of the EOS and Gibbs free energy at $P=0.3621>P_{c}$. For reference, the isobaric curves at $P=P_{c}$ are also depicted in dashed line. Marked points on the left and right diagrams are in one-to-one correspondence
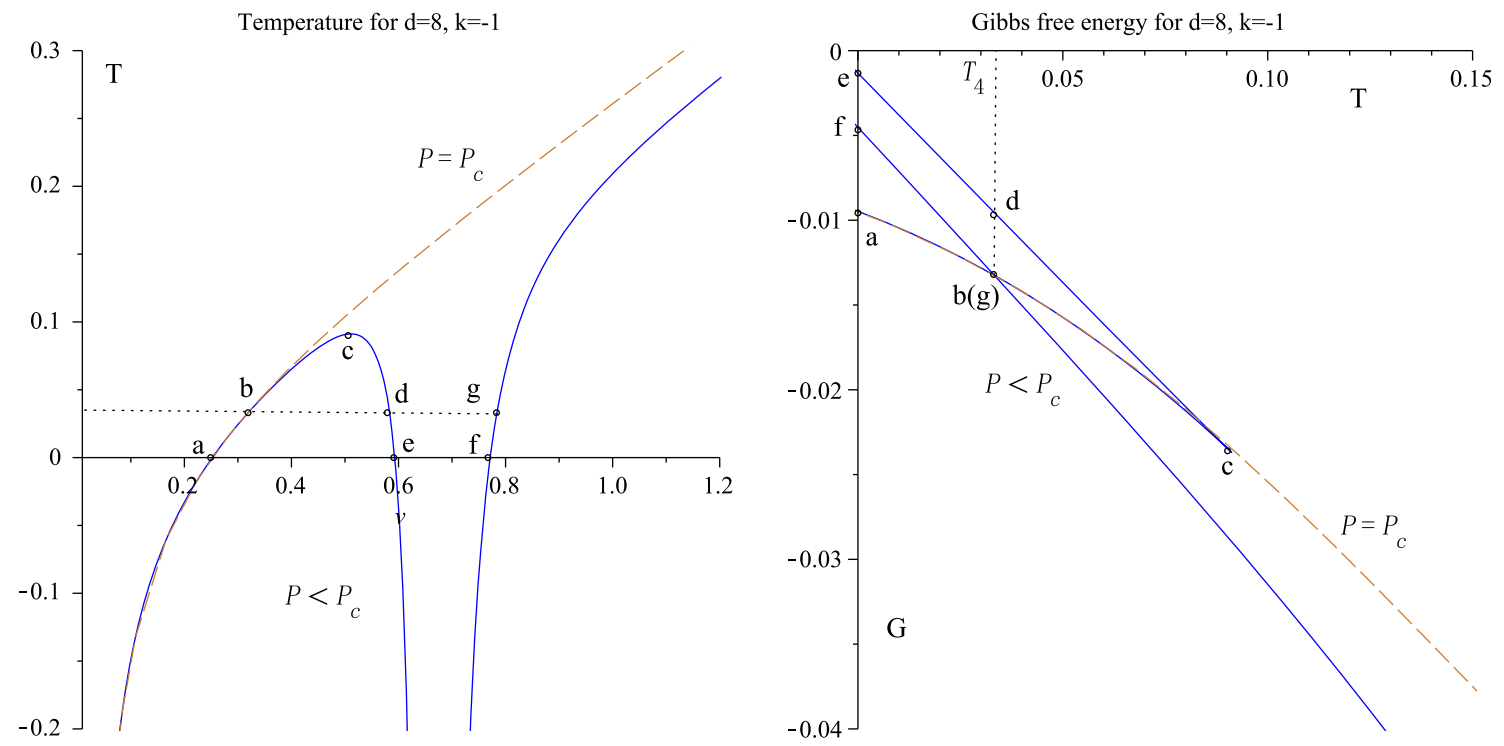

Fig. $10 d=8$ and $k=-1$ : isobaric plots of the EOS and Gibbs free energy at $P=0.2618<P_{c}$. For reference, the isobaric curves at $P=P_{c}$ are also depicted in dashed line. Marked points on the left and right diagrams are in one-to-one correspondence

and $P<P_{c}$ in Figs. 2, 3, 4. Figure 2 is a typical case with $P>P_{c}$. It can be seen that, at the temperature $T_{1}$, there can be three different black hole phases with different $v$, among these, the "small black hole" marked with the letter $\mathrm{b}$ and the "large black hole" marked with $\mathrm{g}$ are both thermodynamically stable, while the "medium sized black hole" marked with e is thermodynamically unstable. The black hole phases $\mathrm{b}$ and $\mathrm{g}$ coexist at temperature $T_{1}$, because these two phases have the same Gibbs free energy. At temperatures lower than the temperature of the black hole state $f$, only the small black hole phase exists, while at temperatures higher than the temperature of the black hole state $\mathrm{g}$, the large black hole phase is thermodynamically favored. The shape of the Gibbs free energy plot can be thought of as containing a swallow tail with one tip extending infinitely long to the right (i.e. the high temperature end). Figures 3 and 4 both correspond to cases $P<P_{c}$. The difference lies in the pressure in Fig. 4, which is even lower than that in Fig. 3, so that in Fig. 3, there still exists a stable small black hole phase, which coexists with the large black hole phase at temperature $T_{2}$, while in Fig. 4, the only stable black hole phase is the large black hole phase ( $T_{2}$ becomes negative). The swallow tails in the Gibbs free energy curves in these two figures are both incomplete, because a small/large portion of the tail 

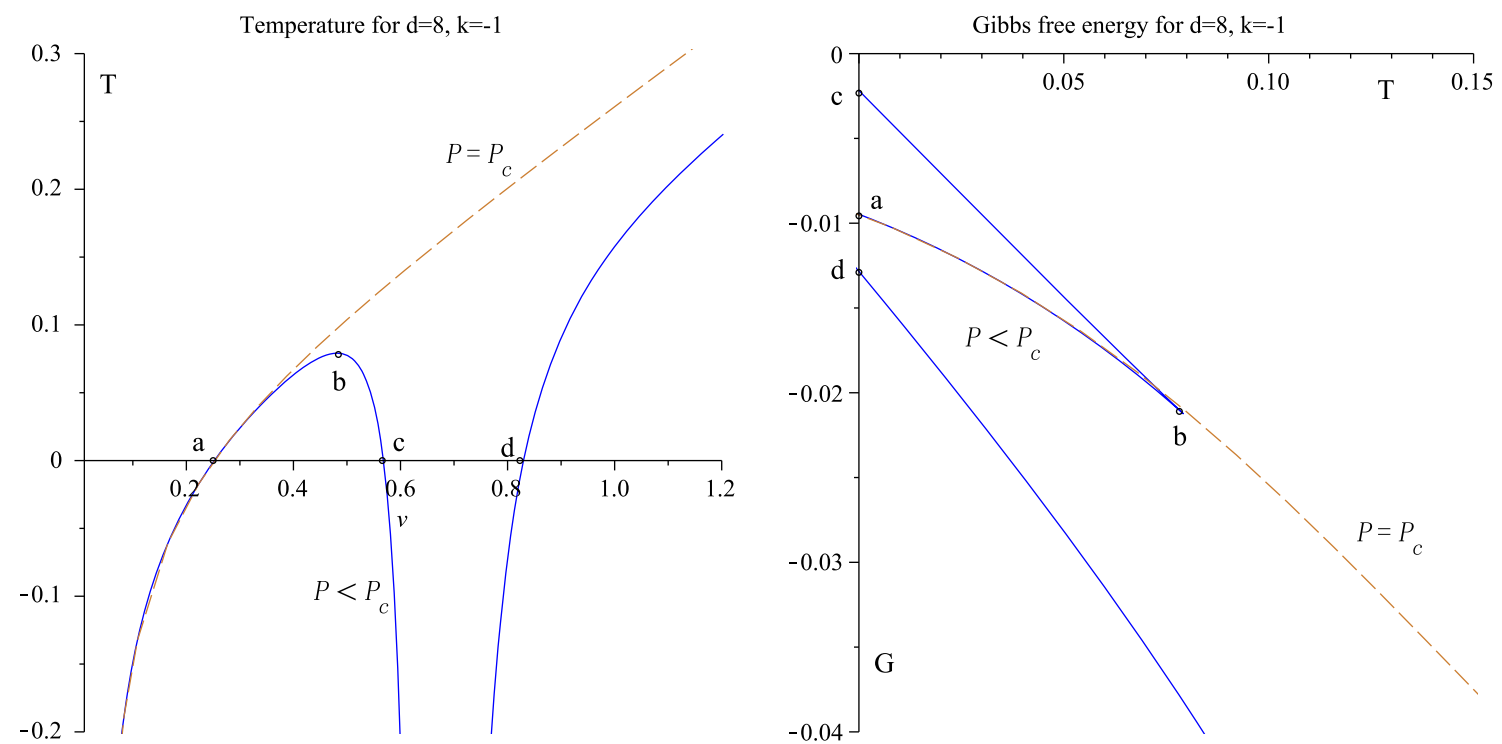

Fig. $11 d=8$ and $k=-1$ : isobaric plots of the EOS and Gibbs free energy at $P=0.2451<P_{c}$. For reference, the isobaric curves at $P=P_{c}$ are also depicted in dashed line. Marked points on the left and right diagrams are in one-to-one correspondence

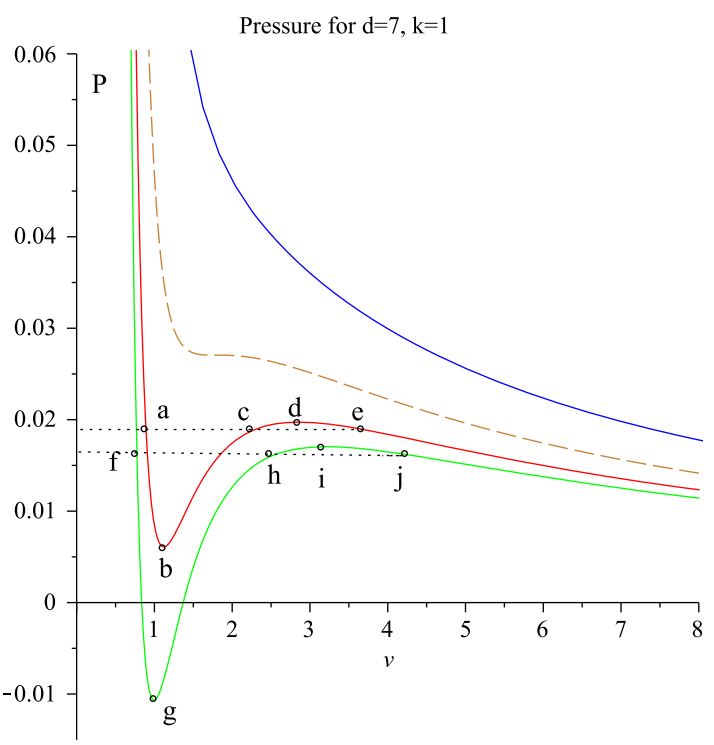

Fig. 12 Isothermal plots of the EOS and Gibbs free energy at $d=7$ and $k=+1$. Each curve corresponds to a constant $T>0$. The $P<0$ region is unphysical. The curves at $T=T_{c}$ are depicted in dashed lines.

extends to the negative temperature axes and hence are cut off from the physical region of the thermodynamical phase space.

Let us now turn to look at the isothermal plots of the EOS and Gibbs free energy. These plots are given in Figs. 5, 6, 7. Figure 5 gives the isothermal plots of the EOS and Gibbs free energy at $T>T_{c}$. It can be seen that at low pressure, the large black hole phase (segment b-g and onwards on the isotherm) is thermodynamically preferred. At high pressure,

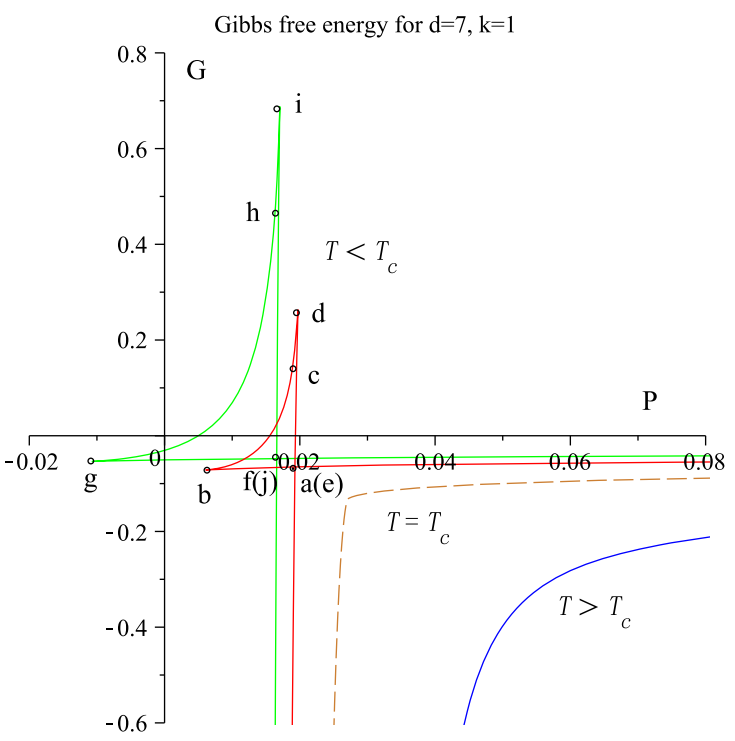

Marked points on the left and right diagrams are in one-to-one correspondence. The apparently straight vertical segments in the Gibbs free energy plots are actually curved, with positive slope everywhere

the small black hole phase (segment b-a and onwards) is thermodynamically preferred. Note that although the Gibbs free energy curve looks like containing a complete swallow tail, the curve is actually discontinuous at the point $\mathrm{c}$. Both Figs. 6 and 7 correspond to the case $T<T_{c}$, the only difference lies in Fig. 7, which corresponds to a temperature even lower than that in Fig. 6, so that a portion of the isobaric curves in Fig. 7 become unphysical (i.e. extend to negative pressure). The phase structure is basically the same as in the case of 


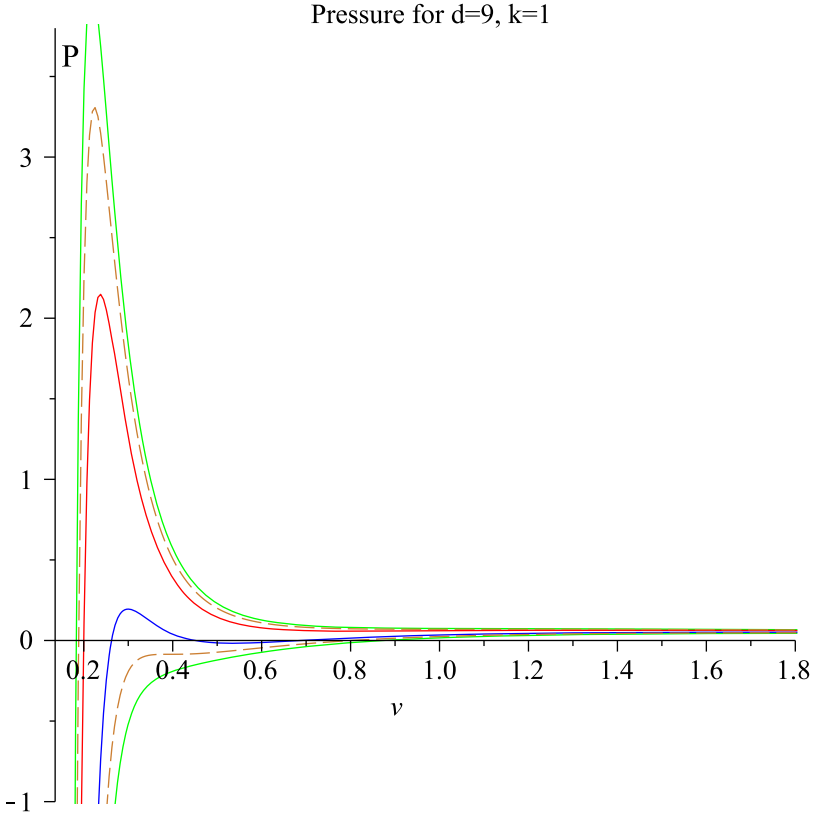

Fig. 13 Isothermal plot of the EOS at $d=9$ and $k=+1$. Each curve corresponds to a constant $T>0$. The dashed lines correspond to $T=T_{c 1}$ and $T=T_{c 2}$. The temperatures decrease from top to bottom

$T>T_{c}$, i.e. large black holes are favored at low pressures and small black holes are favored at high pressures.

\subsubsection{The cases of $d>7$}

When $d>7$, the last term in (9) dominates at small $v$, which results in a significant difference as compared to the $d=$ 7 case. Apart from this, there is no qualitative difference between the different dimensions if they are all above seven.
The best way to illustrate the difference from the case of $d=7$ is via a plot of the EOS. Figure 8 gives the isobaric and isothermal plots of the EOS at $d=8$, which is in analogy to Fig. 1 for the $d=7$ case. The most significant difference from Fig. 1 lies in the fact that all isobaric curves tend to $T \rightarrow-\infty$ as $v \rightarrow 0$. However, there is no qualitative difference in the isothermal curves at positive $T$.

The phase structure for the $d>7$ cases can be worked out exactly as in the $d=7$ case. The resulting phase structure is extremely similar to the $d=7$ case, the only difference lies in the fact that at low pressure, the thermodynamically favored small black hole phase cannot become arbitrarily small; there always exists a smallest $v_{a} \neq 0$, which corresponds to a zero temperature small black hole. If $v$ goes even smaller, the temperature becomes negative, which indicates that the corresponding small black hole becomes unstable and cannot exist physically. For illustrative purposes, we present the isobaric plots for the EOS and Gibbs free energy for $d=8$, $k=-1$ black holes in Figs. 9, 10, 11. These plots are created in complete analogy to Figs. 2, 3, 4. The isothermal plots analogous to Figs. 5, 6, 7 for the $d=8$ case do not reveal any further novelty or difference from the $d=7$ case, so we omit these plots.

\subsection{Spherical case with $k=+1$}

Now we consider the case of $k=+1$. When $d=7$, the last term in the EOS (25) vanishes, the dominating term at small $v$ is the second last term, making $P \rightarrow+\infty$ as $v \rightarrow 0$ (for positive constant $T$ ). In contrast, when $d>7$, the last term in (25) dominates at small $v$, making $P \rightarrow-\infty$ as $v \rightarrow 0$. Therefore, it is still necessary to distinguish the case of $d=7$ from the $8 \leq d \leq 11$ cases (the cases $d>11$ are excluded because there is no critical point in such cases).
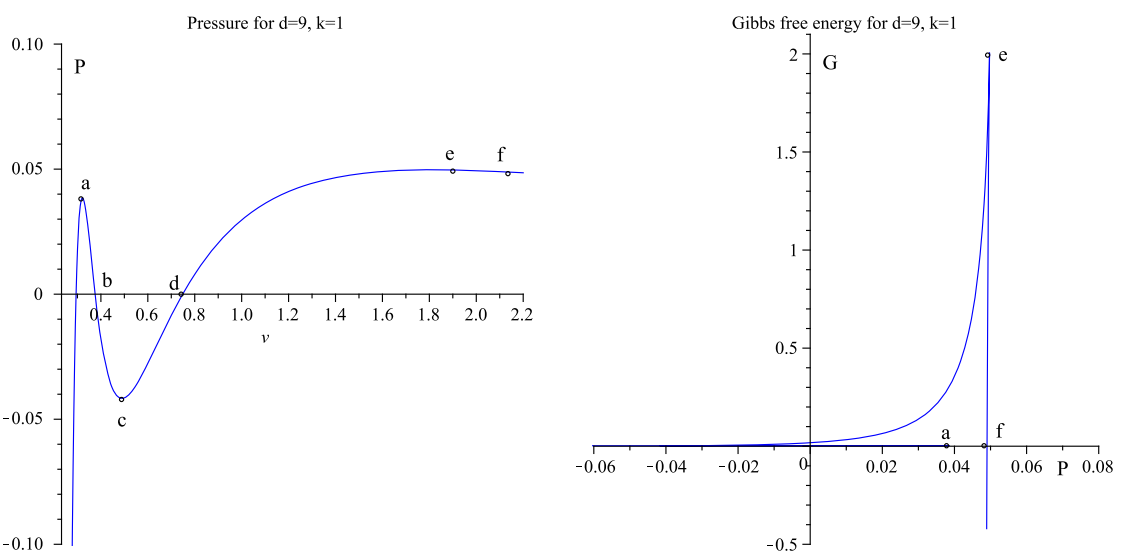

Fig. 14 Isothermal plots of the EOS and Gibbs free energy at $d=9$ and $k=+1$ at $T_{c 1}<T=0.2075<T_{c 2}$. Marked points on the $P-V$ and $G-P$ diagrams are in one-to-one correspondence. The third plot is a magnification of the $G-P$ diagram given in the middle plot. The

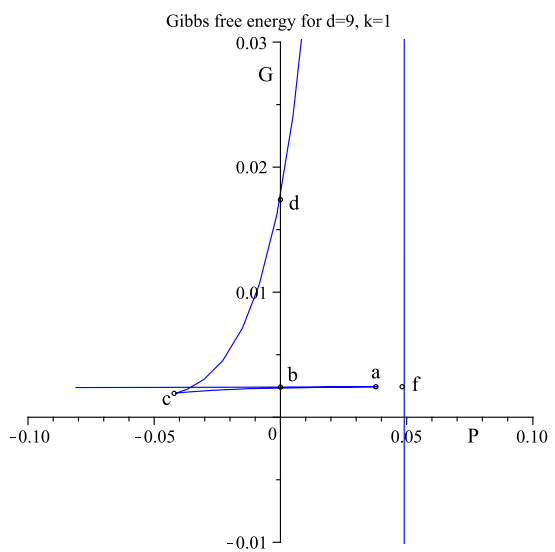

$P<0$ region is unphysical. In this case, there is no first-order phase transition in the $P>0$ region (the Gibbs free energy on the $e-f$ segment and onward is lower than its values on any other segment of the EOS plot) 

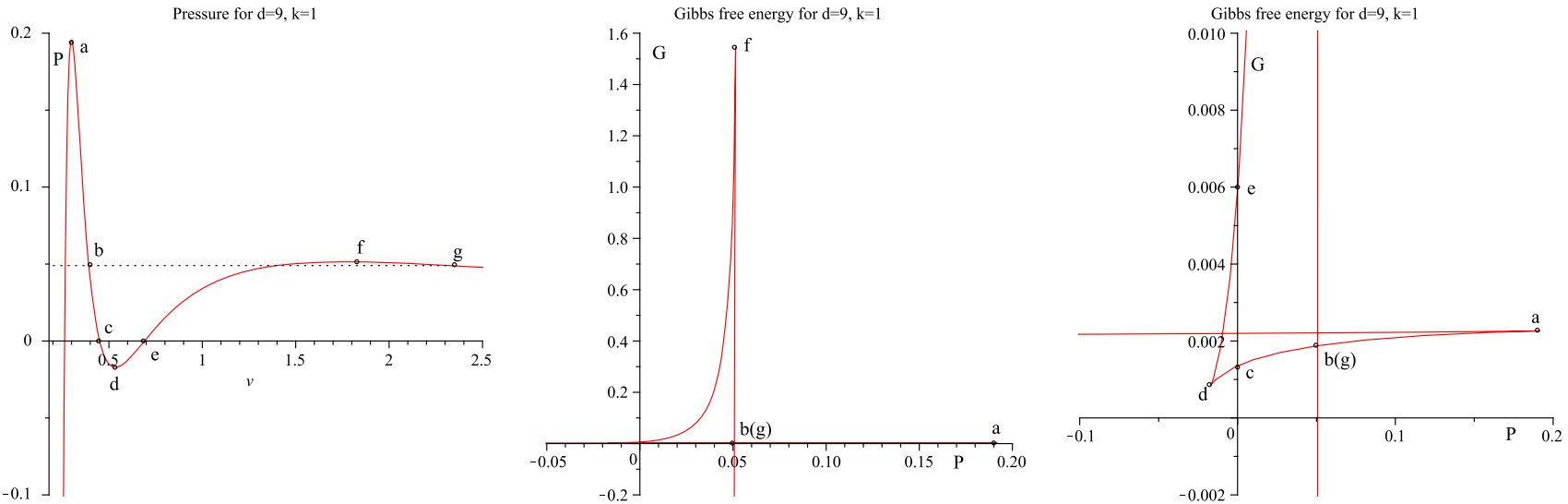

Fig. 15 Isothermal plots of the EOS and Gibbs free energy at $d=9$ and $k=+1$ at $T_{c 1}<T=0.2100<T_{c 2}$. Marked points on the $P-V$ and $G-P$ diagrams are in one-to-one correspondence. The third plot is a magnification of the $G-P$ diagram given in the middle plot. The

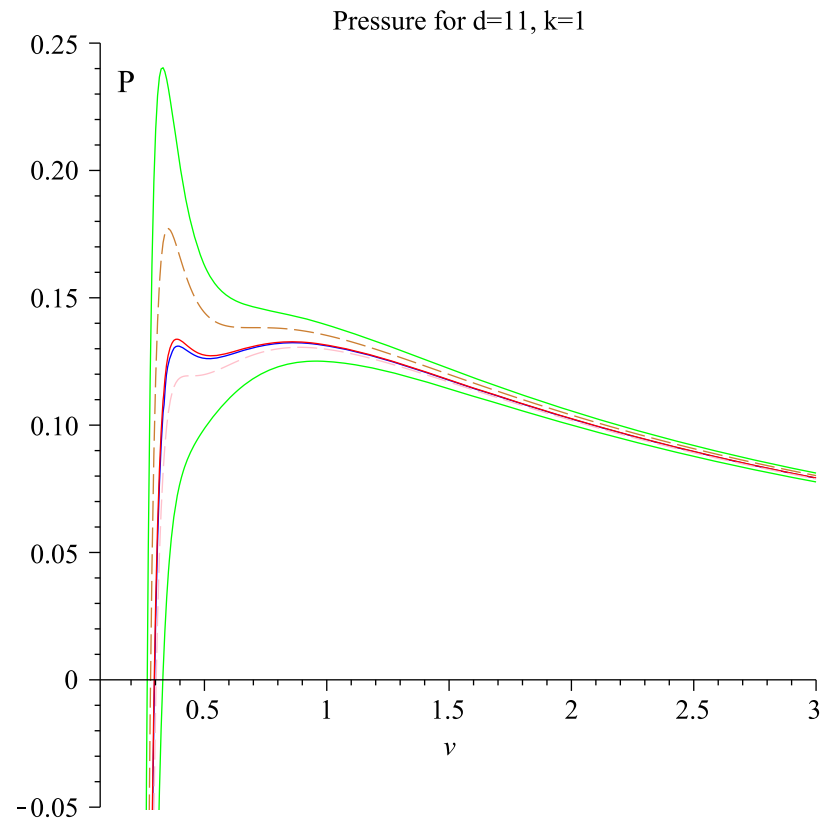

Fig. 16 Isothermal plots of the EOS at $d=11$ and $k=+1$. The temperatures decrease from top to bottom. The dashed lines correspond to $T=T_{c 1}$ and $T=T_{c 2}$. The middle two lines correspond to $T=$ 0.3193 and $T=0.3195$, respectively

\subsubsection{The case of $d=7$}

First we consider the case of $d=7$. As indicated in the last section, there is a single critical point in this dimension. Figure 12 gives the isothermal plots for the EOS and the Gibbs free energy at $k=+1$ and $d=7$. It can be seen that at $T>T_{c}$ (here $T_{c}=T_{c 2}$ is the only critical temperature in $d=7$ ), there is only a single phase which is in analogy to the thermal behavior of an ideal gas. At $T<T_{c}$, multiple
$P<0$ region is unphysical. In this case, there is a first-order phase transition at the marked point $\mathrm{b}(g)$ where the Gibbs free energy degenerate but not differentiable

phases begin to appear. It is remarkable that for sufficiently low temperature $T$, there can be a segment in the isotherms which corresponds to negative pressure $P$. We consider it as unphysical.

For $P>0$, there can be up to three black hole phases at the same pressure, among these, the small black hole phase is favored at high pressure, while the large black hole phase is favored at low pressure. The two thermodynamically favored black hole phases can coexist at some intermediate pressure, while the intermediately sized black hole phase is always thermodynamically unfavored.

\subsubsection{The cases of $8 \leq d \leq 11$}

Now we investigate the case of $8 \leq d \leq 11$ by analyzing the isothermal plots of EOS and Gibbs free energy. In these dimensions there are always two critical points. In $d=8,9$, the critical pressure $P_{c 1}$ becomes negative, which is unphysical. In $d=10,11, P_{c 1}$ remains positive. We shall consider the case of $d=8,9$ and $d=10,11$ separately.

First we consider the cases $d=8,9$. These two cases are not qualitatively different from each other, and so, without loss of generality, we only present the detailed analysis in $d=9$. The two critical pressures are $P_{c 1}=-0.0856$ and $P_{c 2}=0.3928$. The critical temperatures are $T_{c 1}=0.2046$ and $T_{c 2}=0.2302$. Figure 13 gives the isothermal plots for the EOS at $k=+1$ and $d=9$, which is quite similar with the $P-V$ diagram of BI-AdS black holes [28]. Regardless of the value of temperature, the pressure goes to negative infinity as $v \rightarrow 0$ and vanishes as $v \rightarrow \infty$. It can be seen that at $T_{c 1}<T<T_{c 2}$, multiple phases begin to appear. Note that in this region of the temperature, each isotherm possesses three extrema, and it will be clear that at each of 

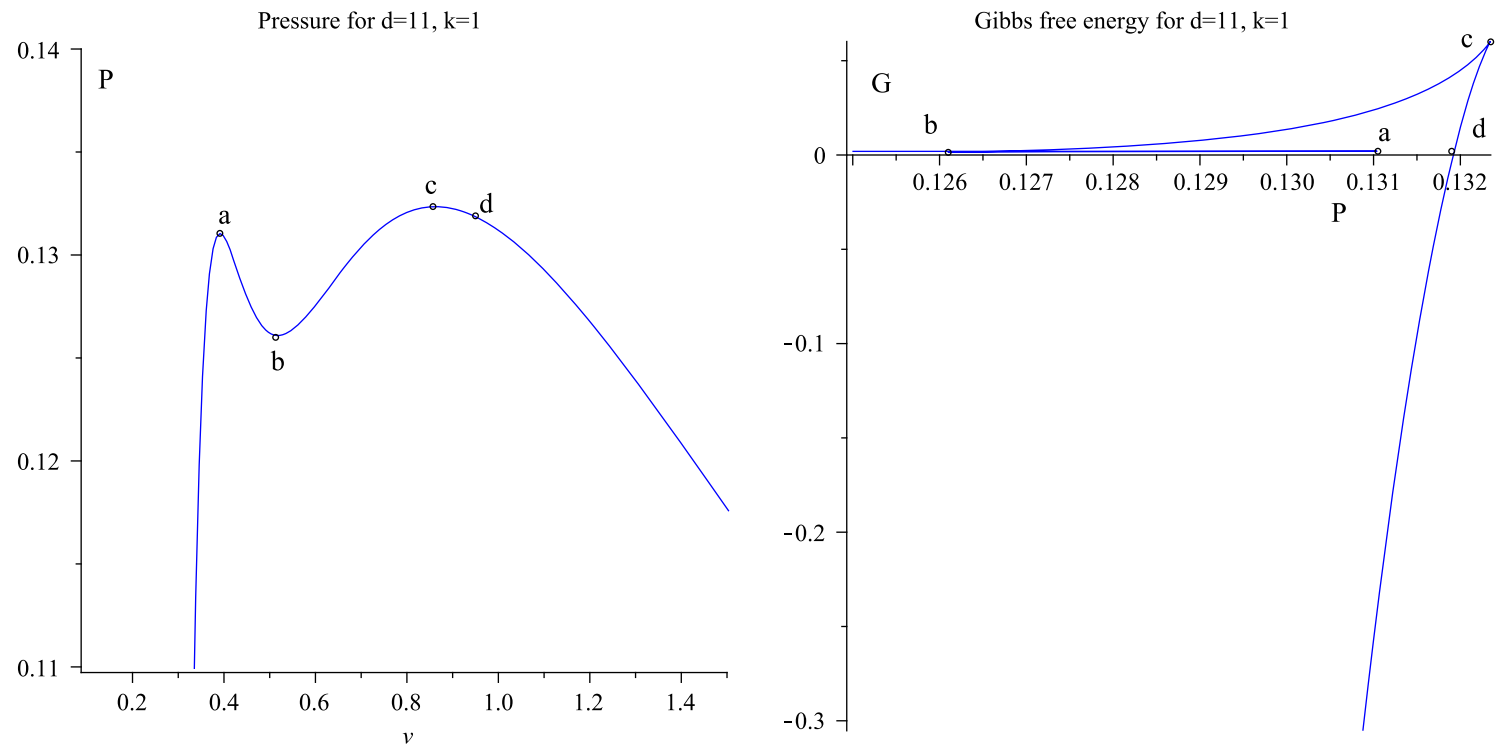

Fig. 17 Isothermal plots of the EOS and Gibbs free energy at $d=11$ and $k=+1$ in $T_{c 1}<T=0.3193<T_{c 2}$. Marked points on the left and right diagrams are in one-to-one correspondence. In this case, there is no phase transition in the region $P>0$

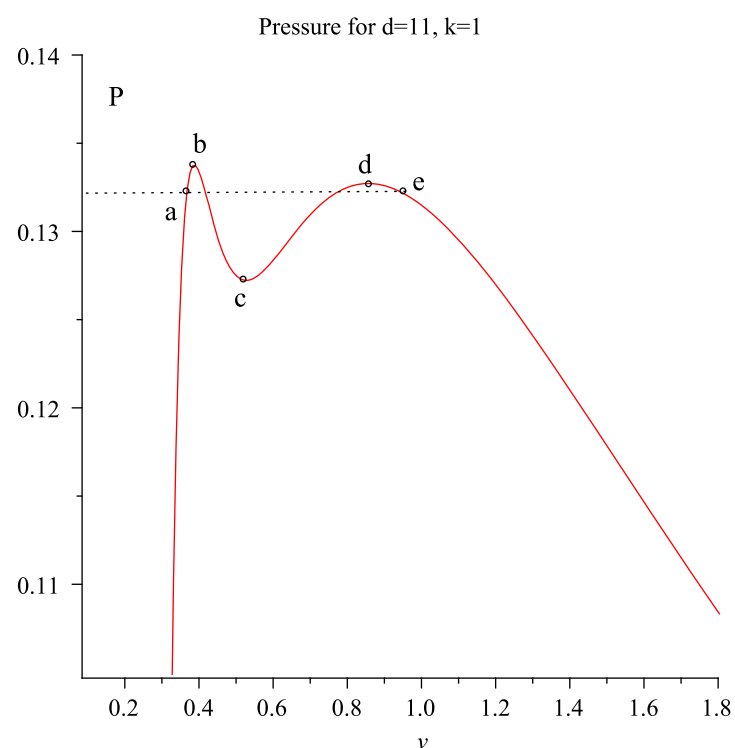

Fig. 18 Isothermal plots of the EOS and Gibbs free energy at $d=11$ and $k=+1$ in $T_{c 1}<T=0.3195<T_{c 2}$. Marked points on the left and right diagrams are in one-to-one correspondence. The global minimum

these extrema the derivative of the Gibbs free energy with respect to the pressure is discontinuous.

In Figs. 14 and 15 we depict two particular temperatures and re-plot the EOS together with the corresponding Gibbs free energy curve. It can be seen from these plots that at some temperature in between $T=0.2075$ and $T=0.2100$ the system begins to develop a first-order phase transition at a particular point in the region $P>0$. However, the exact value of the temperature at which the phase transi-

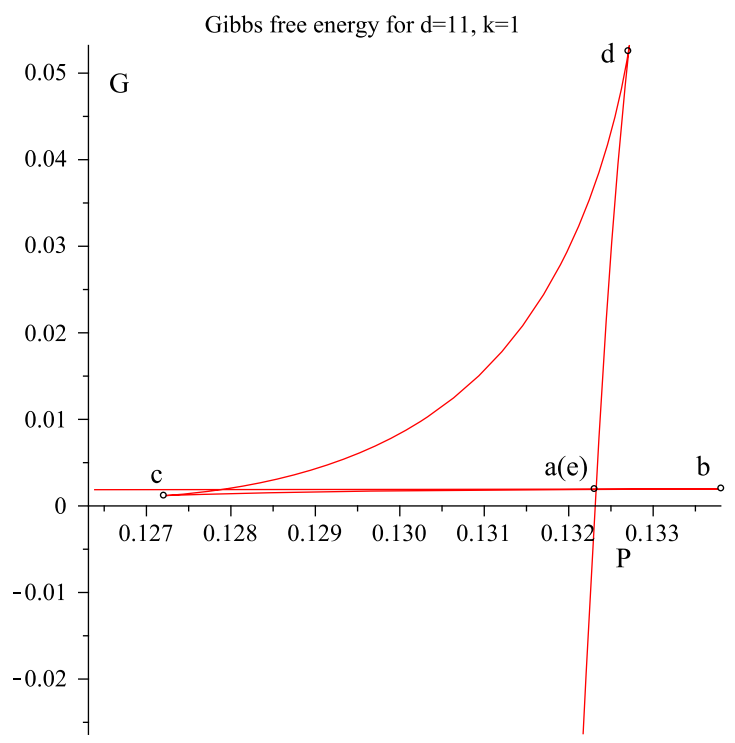

of the Gibbs free energy proves there is a coexistence state of small (point $a$ ) and larger (point $e$ ) black holes

tion begins to appear is very difficult to determine. When the first-order phase transition point is developed, there is also some possibility for the existence of a zeroth-order phase transition in the region $P>0$, which occurs when the pressure at the marked point $\mathrm{f}$ is higher than it is at the marked point a in Fig. 15. We consider the region of $P<0$ as unphysical.

Next we consider the cases of $d=10,11$. These phase structures in two dimensions are qualitatively similar, so we 
take $d=11$ as an example. The critical pressures are $P_{c 1}=$ 0.1194 and $P_{c 2}=0.1385$, respectively, both are positive. The critical temperatures are $T_{c 1}=0.3183$ and $T_{c 2}=0.3221$.

In Fig. 16 we present the isothermal plots for the EOS in $d=11$ and $k=+1$. Multiple phases can appear at temperatures $T$ in between $T_{c 1}$ and $T_{c 2}$. Unlike the cases $d=8,9$, both $P_{c 1}$ and $P_{c 2}$ are positive and physical, so one does not need to take care of the negative pressure region. In analogy to Figs. 14 and 15, we depict the EOS and $G-P$ diagrams in Figs. 17 and 18, with the former corresponding to the cases in when no first-order phase transition occurs and the latter to the cases when a first-order phase transition develops in the $P>0$ region. In the latter case, if the pressure at the point $d$ exceeds that at the point $b$, there is a possibility for the existence of a zeroth-order phase transition in the region $P>0$.

\section{Concluding remarks}

Although the extended phase space thermodynamics for the third-order Lovelock gravity has been studied in some previous works, e.g. [31,41], exploring the thermodynamic phase space in different spacetime dimensions reveals unexpected rich phase structures, which are overlooked in those works.

In this paper we explored the phase structures for the third-order Lovelock gravity in diverse dimensions. Special emphasis is put on the dependence on the spacetime dimensions as well as on the spatial curvature of the black hole horizons. Our work extends that of [31] in that all spacetime dimensions which admit the existence of critical points are worked out, and in that the case $k=-1$ is explored in much more detail. The more recent work [41] studied the phase structures of Lovelock-Born-Infeld gravity and did consider the dependence on the spacetime dimensions. However, the authors of that paper worked out only a single critical point and erroneously described $d=12$ with $k=1$ as a dimension allowing for the existence of critical point. Our work indicates that when $k=1$, only the dimensions $7 \leq d \leq 11$ allow for the existence of critical points.

Open Access This article is distributed under the terms of the Creative Commons Attribution License which permits any use, distribution, and reproduction in any medium, provided the original author(s) and the source are credited.

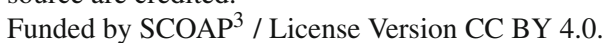

\section{References}

1. S. Hawking, D.N. Page, Thermodynamics of Black Holes in antiDe Sitter Space. Commun. Math. Phys. 87, 577 (1983)
2. A. Chamblin, R. Emparan, C. Johnson, R. Myers, Charged AdS black holes and catastrophic holography. Phys. Rev. D 60, 064018 (1999). arXiv:hep-th/9902170

3. A. Chamblin, R. Emparan, C.V. Johnson, R.C. Myers, Holography, thermodynamics and fluctuations of charged AdS black holes. Phys. Rev. D 60, 104026 (1999). arXiv:hep-th/9904197

4. Y. Liu, D. -C. Zou, B. Wang, Signature of the Van der Waals like small-large charged AdS black hole phase transition in quasinormal modes. arXiv:hep-th/1405.2644

5. D. Kastor, S. Ray, J. Traschen, Enthalpy and the Mechanics of AdS Black Holes. Class. Quant. Grav. 26, 195011 (2009). arXiv:0904.2765

6. B.P. Dolan, The cosmological constant and the black hole equation of state. Class. Quant. Grav. 28, 125020 (2011). arXiv:1008.5023

7. B.P. Dolan, Pressure and volume in the first law of black hole thermodynamics. Class. Quant. Grav. 28, 235017 (2011). arXiv: 1106.6260

8. B.P. Dolan, Compressibility of rotating black holes. Phys. Rev. D 84, 127503 (2011). arXiv: 1109.01980

9. D. Kubiznak, R.B. Mann, P-V criticality of charged AdS black holes. JHEP 1207, 033 (2012). arXiv: 1205.0559

10. G.W. Gibbons, R. Kallosh, B. Kol, Moduli, scalar charges, and the first law of black hole thermodynamics. Phys. Rev. Lett. 77, 4992 (1996). arXiv:hep-th/9607108

11. J.D.E. Creighton, R.B. Mann, Quasilocal thermodynamics of dilaton gravity coupled to gauge fields. Phys. Rev. D 52, 4569 (1995). arXiv:gr-qc/9505007

12. D.A. Rasheed, Nonlinear electrodynamics: Zeroth and first laws of black hole mechanics. arXiv:hep-th/9702087

13. M. Cvetic, G.W. Gibbons, D. Kubiznak, C.N. Pope, Black hole enthalpy and an entropy inequality for the thermodynamic volume. Phys. Rev. D 84, 024037 (2011). arXiv:1012.2888

14. A. Belhaj, M. Chabab, H. El Moumni, M.B. Sedra, On thermodynamics of AdS black holes in arbitrary dimensions. Chin. Phys. Lett. 29, 100401 (2012). arXiv: 1210.4617

15. E. Spallucci, A. Smailagic, Maxwell's equal area law for charged Anti-deSitter black holes. Phys. Lett. B 723, 436 (2013). arXiv: 1305.3379

16. M.B.J. Poshteh, B. Mirza, Z. Sherkatghanad, Phase transition, critical behavior, and critical exponents of Myers-Perry black holes. Phys. Rev. D 88, 024005 (2013). arXiv: 1306.4516

17. A. Belhaj, M. Chabab, H.E. Moumni, L. Medari, M.B. Sedra, The thermodynamical behaviors of Kerr-Newman AdS black holes. Chin. Phys. Lett. 30, 090402 (2013). arXiv:1307.7421

18. N. Altamirano, D. Kubiznak, R.B. Mann, Z. Sherkatghanad, KerrAdS analogue of tricritical point and solid/liquid/gas phase transition. arXiv: 1308.2672

19. N. Altamirano, D. Kubiznak, R.B. Mann, Reentrant phase transitions in rotating AdS black holes. Phys. Rev. D 88, 101502 (2013). arXiv: 1306.5756

20. N. Altamirano, D. Kubiznak, R.B. Mann, Z. Sherkatghanad, Thermodynamics of rotating black holes and black rings: phase transitions and thermodynamic volume. arXiv:1401.2586

21. S.-W. Wei, Y.-X. Liu, Critical phenomena and thermodynamic geometry of charged Gauss-Bonnet AdS black holes. Phys. Rev. D 87(4), 044014 (2013). arXiv:1209.1707

22. R.-G. Cai, L.-M. Cao, L. Li, R.-Q. Yang, P-V criticality in the extended phase space of Gauss-Bonnet black holes in AdS space. JHEP 1309, 005 (2013). arXiv: 1306.6233

23. D.-C. Zou, Y. Liu, B. Wang, Critical behavior of charged Gauss-Bonnet AdS black holes in the grand canonical ensemble. arXiv: 1404.5194

24. S. Chen, X. Liu, C. Liu, J. Jing, $P-V$ criticality of AdS black hole in $f(R)$ gravity. Chin. Phys. Lett. 30, 060401 (2013). arXiv:1301.3234 
25. K. Hristov, C. Toldo, S. Vandoren, Phase transitions of magnetic AdS4 black holes with scalar hair. Phys. Rev. D 88, 026019 (2013). arXiv: 1304.5187

26. A. Belhaj, M. Chabab, H. El Moumni, M. B. Sedra, Critical behaviors of 3D black holes with a scalar hair. arXiv:1306.2518

27. S.H. Hendi, M.H. Vahidinia, P-V criticality of higher dimensional black holes with nonlinear source. Phys. Rev. D 88, 084045 (2013). arXiv: 1212.6128

28. S. Gunasekaran, R.B. Mann, D. Kubiznak, Extended phase space thermodynamics for charged and rotating black holes and Born-Infeld vacuum polarization. JHEP 1211, 110 (2012). arXiv:1208.6251

29. D.-C. Zou, S.-J. Zhang, B. Wang, Critical behavior of Born-Infeld AdS black holes in the extended phase space thermodynamics. Phys. Rev. D 89, 044002 (2014). arXiv:1311.7299

30. M.-S. Ma, H.-H. Zhao, L.-C. Zhang, R. Zhao, Existence condition and phase transition of Reissner-Nordström-de Sitter black hole. arXiv:1312.0731

31. J.-X. Mo, W.-B. Liu, P-V Criticality of topological black holes in Lovelock-Born-Infeld gravity. Eur. Phys. J. C 74, 2836 (2014). arXiv: 1401.0785

32. W. Xu, H. Xu, L. Zhao, Gauss-Bonnet coupling constant as a free thermodynamical variable and the associated criticality. Eur. Phys. J. C 74, 2970 (2014). arXiv:1311.3053

33. R. Banerjee, S. Ghosh, D. Roychowdhury, New type of phase transition in Reissner Nordstrom-AdS black hole and its thermodynamic geometry. Phys. Lett. B 696, 156 (2011). arXiv:1008.2644
34. A. Lala, D. Roychowdhury, Ehrenfest's scheme and thermodynamic geometry in Born-Infeld AdS black holes. Phys. Rev. D 86, 084027 (2012). arXiv:1111.5991 [gr-qc]

35. A. Lala, Adv. High Energy Phys. 2013, 918490 (2013). arXiv: 1205.6121 [gr-qc]

36. B.R. Majhi, D. Roychowdhury, Class. Quant. Grav. 29, 245012 (2012). arXiv:1205.0146 [gr-qc]

37. M.H. Dehghani, M. Shamirzaie, Thermodynamics of asymptotic flat charged black holes in third order Lovelock gravity. Phys. Rev. D 72, 124015 (2005). arXiv:0506227 [hep-th]

38. M.H. Dehghani, R. Pourhasan, Thermodynamic instability of black holes of third order Lovelock gravity. Phys. Rev. D 79, 064015 (2009). arXiv:0903.4260

39. D. Zou, R. Yue, Z. Yang, Thermodynamics of third order Lovelock anti-de Sitter black holes revisited. Commun. Theor. Phys. 55, 449 (2011). arXiv:1011.2595

40. D. Kastor, S. Ray, J. Traschen, Smarr formula and an extended first law for Lovelock gravity. Class. Quant. Grav. 27, 235014 (2010). arXiv: 1005.5053

41. A. Belhaj, M. Chabab, H.E. Moumni, K. Masmar, M.B. Sedra, Ehrenfest scheme of higher dimensional topological AdS black holes in Lovelock-Born-Infeld gravity. arXiv:1405.3306 